\title{
Identifying problems associated with focus and context awareness in 3D modelling tasks
}

\author{
Masood Masoodian ${ }^{1}$ And Azmi Bin Mohd Yusof $^{2}$ And Bill \\ ROGERS $^{1}$ \\ ${ }^{1}$ Department of Computer Science, The University of Waikato, New Zealand \\ ${ }^{2}$ Faculty of Computer Science, Universiti Tenaga Nasional, Malaysia
}

\begin{abstract}
Creating complex 3D models is a challenging process. One of the main reasons for this is that 3D models are usually created using software developed for conventional 2D displays which lack true depth perspective, and therefore do not support correct perception of spatial placement and depth-ordering of displayed content. As a result, modellers often have to deal with many overlapping components of $3 \mathrm{D}$ models (e.g. vertices, edges, faces, etc.) on a $2 \mathrm{D}$ display surface. This in turn causes them to have difficulties in distinguishing distances, maintaining position and orientation awareness, etc. To better understand the nature of these problems, which can collectively be defined as "focus and context awareness" problems, we have conducted a pilot study with a group of novice 3D modellers, and a series of interviews with a group of professional 3D modellers. This article presents these two studies, and their findings, which have resulted in identifying a set of focus and context awareness problems that modellers face in creating $3 \mathrm{D}$ models using conventional modelling software. The article also provides a review of potential solutions to these problems in the related literature.
\end{abstract}

Categories and subject descriptors: Graphics systems and interfaces; Empirical studies in HCI; user studies; field studies

Keywords: 3D modelling; focus and context awareness; visualization; user studies; interviews

Responsible Editorial Board Member: Editorial Board member name

\section{INTRODUCTION}

Advances in computer technology, in terms of faster processing power and increased memory capacity, have made it possible to create and render highly realistic 3D models. Despite these, the process of creating 3D models has remained largely unchanged since the 1990's. Although these days there are many techniques for creating 3D models (e.g. based on solid geometry, point cloud, 3D scanning, sketching, etc.) polygon-based 3D modelling is still the most commonly used technique. Modern 3D modelling software such as Maya and 3ds Max [Autodesk, 2014] provide a large range of functions to assist modellers (i.e. their users) with creating, editing, and rendering polygonal 3D models. These tasks, however, remain challenging and cumbersome.

Difficulties faced by 3D modellers can be generally divided into two categories. The first category is related to the problem of having to learn the large set of complex commands and functions which most 3D modelling software offer (i.e. system control). These can be overcome through practice, and over time a user can learn and remember hundreds of commands, shortcuts, etc.

The second category of challenges is related to learning to operate in a $3 \mathrm{D}$ world which is projected on to a $2 \mathrm{D}$ computer display surface. As 3D models become more complex, the user of a 3D modelling software has to cope with an increasing number of overlapping vertices, edges, 
and faces. Although modellers learn to somehow manage this group of challenges, they may never overcome them completely.

More effective 3D modelling tools and visualization techniques are therefore needed to specifically address the problems associated with creating 3D models using conventional 2D display technology. Before such techniques can be developed however, it is important to better understand the extent of these types of problems. In this article we identify a particular range of problems, which we define as the focus and context awareness problems. We then investigate how modellers deal with these problems using existing polygonal 3D modelling software. It should be noted that there may also be other types of problems besides those relating to focus and context awareness. These are however beyond the scope of our investigations as reported in this article.

Here we describe a pilot study of novice modellers we carried out to investigate the range of problems they face when using conventional 3D modelling software. The findings of this study were then used to guide the design of a more comprehensive study which focused on professional 3D modellers. This study consisted of a series of interviews and observations, and investigated the kinds of focus and context awareness issues that professional modellers face. Figure 1 presents a diagrammatic summary of the studies presented in this article.

The article starts by providing an overview of focus and context awareness in 3D modelling tasks (Section 2). This is followed by describing the pilot study of the novice modellers (Section 3), and its findings (Section 3.3). We then present the study of the professional 3D modellers (Section 4) which has resulted in identifying a set of four related focus and context awareness problems (Section 4.5). Finally, the article discusses how these problems may be addressed by future software tools, by reviewing potential solutions from related research (Section 5), and presents some conclusions (Section 6).

\section{FOCUS AND CONTEXT AWARENESS IN 3D MODELLING TASKS}

Nunnari and Simone [2004] define focus as a center of interest or activity, and note that focus is "characterized by a high degree of user involvement to govern the flow of tasks, and is devoted to supporting users in accomplishing their individual or shared tasks". Thus, the success of a task is dependent on users' ability to recognize their center of interest throughout the task, and isolating it from the rest of the non-focus area can increase their efficiency in performing the task [Kosara et al., 2002].

The term context, on the other hand, refers to information other than the object of interest that is visible within the field of vision. Context is often perceived as the extra information, which is not directly relevant to most of the action being carried out on the point of interest, but is referred to by the user in an indirect and occasional manner. Schilit et al. [1994] note that context is more than just knowledge, because it often involves other things that are of interest to the user (i.e. focus) which may constantly change.

The term awareness originates from the field of Gestalt therapy [Yontef, 1993], and has since been commonly used in Computer Supported Cooperative Work. In group work, awareness refers to an understanding of the activities of others, which provides a context for each individual's own activities [Dourish and Bellotti, 1992]. Various tools have therefore been proposed to support better group awareness, mainly in 2D environment [Gutwin et al., 1996, Gutwin and Greenberg, 1998].

Similarly, Focus and context awareness has also been investigated by a number of researchers. For instance, Yeh and Wickens [2001] conducted an experiment to determine how focus and context awareness is used in map reading tasks. They asked the participants to answers questions about information displayed to them. Although the participants were initially asked to answer questions with less visible information (i.e. context) being displayed, they were able to view the context whenever necessary. The results showed that participants often re-displayed or turned on the hidden information, even though it was not directly related to the tasks they were performing. The study demonstrated that the participants felt less comfortable when less information was displayed, and this affected their ability to give correct answers.

Khedr [2004] points out that the awareness created from task-relevant information is helpful. The information however needs to be relevant (i.e. available when needed), and information overload should be avoided, as processing unnecessary information causes distraction.

As mentioned earlier, polygonal 3D modelling tasks are usually done using one of the many existing $3 \mathrm{D}$ modelling software. These systems have generally been developed for conventional 2D displays, and as such, project the 3D modelling world and its objects on to one or more 2D projection surfaces (called viewports), each of which is a perspective or orthogonal view of the $3 \mathrm{D}$ world. $3 \mathrm{D}$ modellers often use multiple computer displays and open several viewports, each with a different view, to have sufficient visual information to carry out their modelling tasks.

Some of these tasks (e.g. vertex selection) require the modeller to have a detailed view of the object or objects they are working on, while others (e.g. object rotation) benefit from having an overview of the entire 3D world, or at least a large part of it. There are, however, many other tasks which require the modeller to simultaneously 


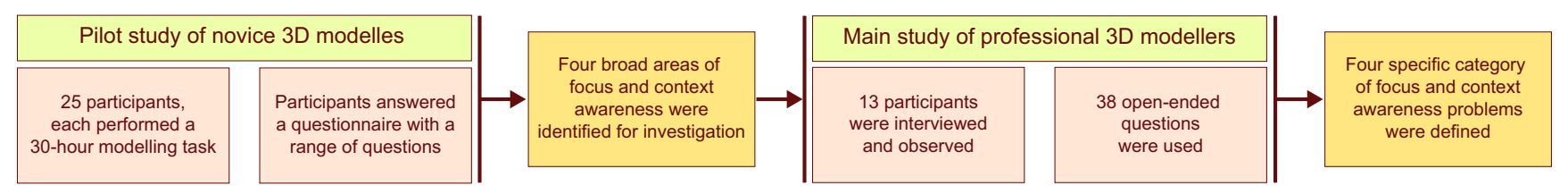

Figure 1. Diagrammatic summary of the studies presented in this article.

be aware of not only the object they are working on (i.e. their focus), but also the larger context in which that object exists. Furthermore, polygonal 3D models often consist of a large number of components, which makes focus and context awareness even more challenging. In such cases focusing on the center of interest within the correspondingly dense data is not always a trivial task, resulting in the viewer having difficulties in locating their point of interest in the sea of data [Krüger and Fogal, 2010].

Dourish [2004] notes that context and activity go hand in hand, and argues that context arises from the navigation and manipulation activities. However, in certain conditions the context that arises from the activity being performed may not be relevant to it, and therefore, this irrelevant context has to be hidden or removed. This situation can apply to $3 \mathrm{D}$ modelling where the processes of navigation and manipulation of the model often change the viewing orientation, resulting in a new context being produced. In some cases context may no longer be helpful to the task in progress, or in fact due to the large amount of visible data context may actually become an obstacle to task performance.

In existing 3D modelling software and hardware environments, modellers tend to cope with these challenging modelling tasks by using techniques such as opening multiple viewports, changing focus to different viewports, zooming in/out, hiding some of the objects, rotating around objects or scenes, and so on. However, even using a combination of these techniques is not always sufficient to provide enough focus and context awareness, and confusion can arise from the failure of the modeller to recognize context while focusing on a specific object. Although addressing the issues related to focus and context awareness is crucial to reducing some of the main challenges of 3D modelling tasks, these issues are rather complex and have not been previously studied in relation to the $3 \mathrm{D}$ modelling process.

\section{PILOT STUDY OF 3D MODELLERS}

We carried out an initial pilot study to broadly identify some of the issues related to the types of problems faced by $3 \mathrm{D}$ modellers while performing their modelling tasks. This exploratory study was questionnaire-based and aimed to investigate the following questions:

- What are some of the potential problems faced by modellers while performing 3D modelling tasks?

- What are the modelling situations that may cause these potential problems to occur?

- How do modellers overcome these problems when they occur using existing 3D modelling software tools?

\subsection{Pilot study participants}

The study participants were undergraduate computer science students taking a course in graphics and multimedia. They were invited to fill out a questionnaire after they had completed a 3D modelling course assignment using Blender [2014].

Blender was the modelling software used in the course the students were taking. It is a commonly used open source polygonal modelling software which provides similar functionality to other modelling software such as MAYA and 3ds Max [Autodesk, 2014].

The students' anonymous participation in this study was totally voluntary and did not contribute to their course grade. This group of students was chosen because they had some basic knowledge of 3D modelling, but were not experts, and were less likely to have bias toward a particular modelling software.

Twenty five students (13 male, 12 female), aged 20-25 years old, took part in this study. None of them considered themselves to be experts in 3D modelling. Thirteen of them had intermediate level expertise in using Blender, while the others were beginners. Sixteen of them had some experience in using MAYA (6 intermediate level, 10 beginners), and 8 had also some experience in using $3 \mathrm{ds}$ Max (all intermediate level).

\subsection{Pilot study task and questionnaire}

The study participants had to create a fully textured skinned character of their own design as part of their 3D modelling coursework.

They were asked to create a 3D polygonal mesh model (with no more than 3000 triangles) of a character which 
could then be used for animation. The modelling task was estimated to take around 30 hours on average to complete, over a 3 -week period. The participants worked in their own time and were not observed while working.

The design of the questionnaire was guided by a review of literature on different $3 \mathrm{D}$ modelling techniques in general, and polygonal modelling in particular. A summary of the questionnaire is presented in Table A1. Questions 1-3 collected demographic data, and questions 4-15 focused on the participants' experience of using Blender to create their 3D models. Questions 7-15 used a 5-point Likert scale to get the participants' ratings of different aspects of the 3D modelling process. These questions also asked the participants to provide free-form textual comments to clarify their ratings.

\subsection{Results of the pilot study}

\subsubsection{Task completion}

Question 4 asked if the participants felt they had completed their assignment successfully. Fifteen participants $(60 \%)$ felt that they had not successfully completed their assignment

(note that this does not mean they did not submit work with each part of the modelling task attempted; just that some felt that they should have done better). One of the reasons given by the participants as to why they believed they had not satisfactorily completed their work was due to the difficulty they said they had in using Blender. This is also reflected in their responses to some of the other questions of the questionnaire, as discussed below.

\subsubsection{Deleting the model and starting over}

Question 5 asked if the participants had ever intentionally deleted their model at some point and started over again with a new model. The aim of this question was to determine the causes of such a drastic action. Sixteen participants (68\%) said they had deleted their model one or more times.

One of the reasons for deleting an object that was mentioned by one of the participants is "because the shape became complex and the vertices were not moving properly to form a shape, and when I only select[ed] one vertex to move, a whole lot of deselected vertices of the other side moved too and ruined the shape". Here, the respondent claims that several unselected vertices were moved, and this ruined the shape of their model. However, clearly an unselected vertex is not affected when other selected vertices are moved or transformed. Therefore, the reason for this claim is probably because the participant did not realise which vertices were selected, due to unintentional selection of overlapping or other close by vertices.

Another reason given for deleting a model and starting over was simply getting lost while performing 3D modelling tasks. This has been identified as an issue by Russo dos Santos et al. [2000], who point out that when users are interacting with a $3 \mathrm{D}$ virtual world, they need to have easy access to information to allow for judicious decision making when solving eventual problems. For the user's movements to be efficient, it is important for the modeller to have a spatial knowledge of the environment and a clear understanding of their location.

\subsubsection{Use of multiple viewports}

In answer to Question 6, all the respondents noted that they often had two or more viewports open while performing their tasks. Generally, the purpose of having more than one viewport open is to enable modellers to view and work on details of the 3D model in one viewport while having an overview or different views of the 3D model in the others.

The respondents' feedback showed that their most commonly used views were the front, top and side views. While in orthographic mode, $15(80 \%)$ of the respondents regularly used the front view, 18 (72\%) used the top view, and $21(84 \%)$ used the side view. In the perspective mode, the numbers were very similar, with $18(72 \%)$ regularly using the front view, 15 (60\%) using the top view, and 15 $(60 \%)$ using the side view.

\subsubsection{Viewing all the objects of the model}

Question 7 asked if the participants had used the view all function or not, and how useful they had found it if they had used it. The view all function in Blender makes all the objects of the model visible to the viewer by zooming and pointing to an appropriate vantage point. As a consequence, the viewing size and position of the objects on the screen is usually altered in order to accommodate them in the viewport.

The analysis of the participants' responses, as shown in Figure 2, indicates that the participants rated the importance of being able to view all the objects of their model above average $(M=3.24, S D=1.23,1$ :not important, 5:very important).

\subsubsection{Realizing the viewer's location}

Question 8 investigated the need for having a virtual eye location indicator on the viewports. The eye location indicator is used in some 3D modelling software to show where the model is being viewed from. For example Doga [2014] uses a red dot to indicate the position of the eye, and blue lines to represent the viewing direction for the perspective view (see Figure 3). However, although the virtual eye location indicator assists the viewer in determining their viewing position, it adds extra visual lines to the viewport, making it even more crowded.

The results of the analysis, as shown in Figure 2 , indicate that many participants found the lack of 


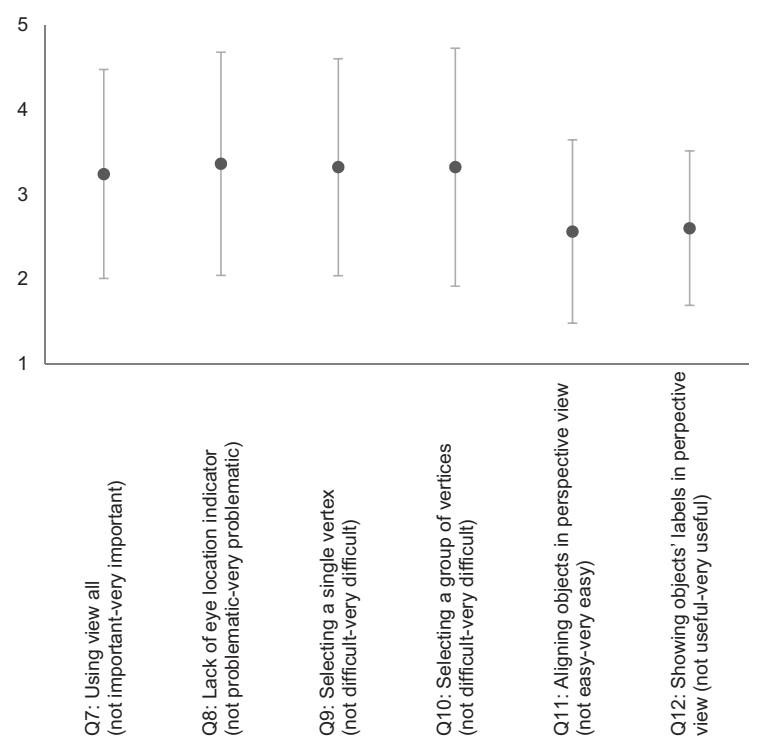

Figure 2. Average ratings for questions $7-12$ of the pilot study questionnaire.

information about the eye location in Blender problematic $(M=3.36, S D=1.32,1$ :not problematic, 5:very problematic).

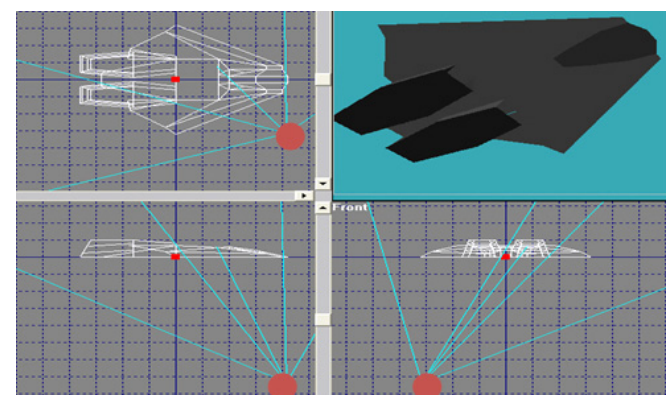

Figure 3. Eye location (red dot with blue lines) in Doga 3D modelling software.

\subsubsection{Selecting a vertex or a group of vertices}

The participants were asked whether or not selecting a single vertex (Question 9) or a group of vertices (Question 10) was an easy task. The results of the analysis, as shown in Figure 2, indicate that the study participants found both types of selection tasks reasonably difficult $(M=3.32$ in both cases, $S D=1.28$ and $S D=1.41$, 1:not difficult, 5:very difficult). The task of selecting a single vertex or a group of vertices is frequently repeated in 3D modelling, and as such being able to perform it with accuracy and ease is critically important.

One of the study participants pointed out that "[I] often need to zoom in and zoom out to understand the model better. In some views it was very hard to see where a particular vertex was, and it took some time to select the correct one because the vertices can be close to each other". This respondent used the zoom-in technique to increase the visibility of the targeted vertex by showing a larger gap between the vertices. Figure 4 illustrates an example of this zooming technique and how it can be helpful. In Figure $4 \mathrm{a}$, vertices $A$ and $B$ are so close to each other that the distance between them is not easily recognized. The distance between them becomes more clear after the model is zoomed-in, as shown in Figure 4b. However, this technique causes some of the model to move out of the viewport.
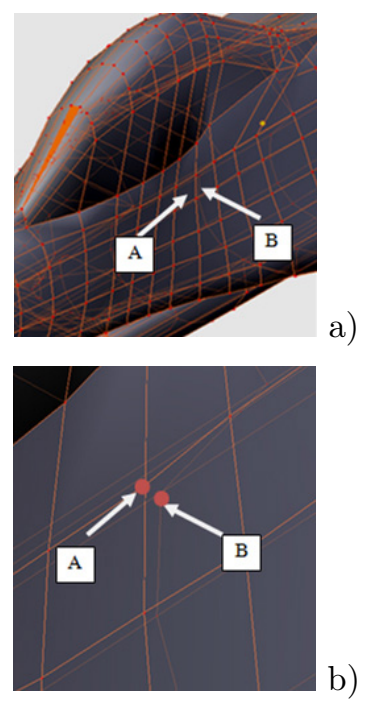

Figure 4. View of the selected vertices (a) before zooming in, and (b) after zooming in.

A second problem related to selecting vertices was highlighted by another participant, "[I] wasn't sure how to select [a] group of vertices. In many instances, I often selected the one I didn't want. You would assume the one in front would be selected but it would choose the one behind". The problem of not being able to select a group of vertices accurately is often caused by the lack of accuracy of the selection tool being used. In Blender, group selection is done by interactively drawing a rectangle around the vertices. However, this tool is not very accurate because it is not able to identify whether the vertices within the rectangle are on the front or back surface of the model. Figure 5 illustrates this problem in Blender. In Figure 5a, a yellow rectangle is drawn, with the aim of selecting vertices of interest $A, B$, and $C$. Figure 5b shows the result of using the rectangle selection tool, where a fourth vertex $D$ belonging to the back face of the model has also been selected. 


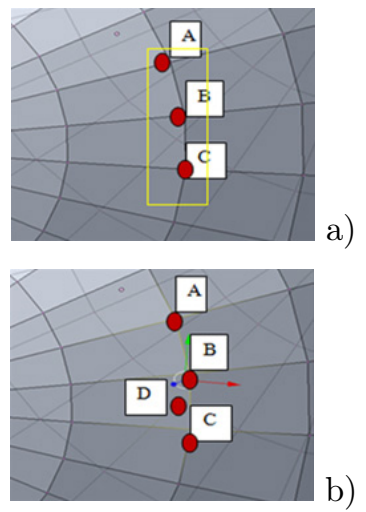

Figure 5. The process of (a) selecting a group of vertices using the block selection, and (b) the result after selection.

A third difficulty of selecting vertices is highlighted by another participant who had to "move [navigate] around the model in order to be sure that the correct component is selected". Figure 6 illustrates an example of this situation, where vertices and edges of the front and back faces of the model are visible. Vertices $A$ and $B$ look near each other when viewed from the direction in Figure 6a. In this example, several vertices are selected (shown in yellow). However, the status of vertex $B$, because of its location on the back face, cannot be identified easily. In order to verify the status of the selection, the modeller would need to navigate around the model. Figure $6 \mathrm{~b}$ verifies that in this example vertex $B$ is actually selected. Although this type of navigating can be used to verify the status of the selected vertices, it can also cause the modeller to lose their focus as they move around the model.
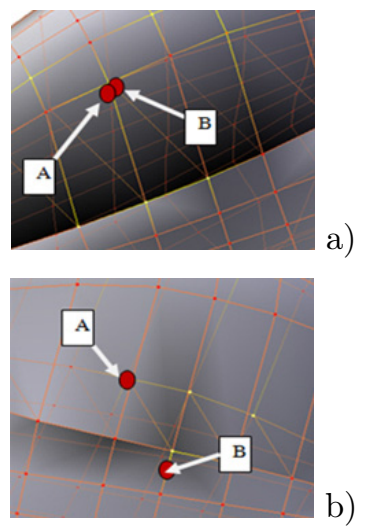

Figure 6. The selected components of a model being viewed form (a) one perspective, and (b) another perspective.

In most cases selection problems occur mainly because 3D modelling software select vertices regardless of their depth order. A better solution, adopted by some modelling software (e.g. ZBrush [Pixologic, 2014]), might be that by default only the vertices located on the front face of the model can be selected by the user.

\subsubsection{Alignment and placement of objects}

Question 11 asked the participants to rate how easy it was to align objects in perspective mode, for example putting an object on top of another. The analysis of the ratings, as shown in Figure 2, suggests that aligning objects is not a particularly easy task $(M=2.56, S D=1.08,1$ :not easy, 5:very easy).

One of the participants referred to a case where "in one viewport the objects looked nicely aligned, but they were not when seeing from another viewport. So I realigned them again but then realized that they were wrong in another viewport". Another participant pointed out that "moving objects for aligning purpose often results [in] the objects being moved far away from the targeted location... it is confusing as you think that they are close or aligned to each other".

Figure 7 illustrates an example of this type of alignment problem, showing how the view provided by a single viewport can be misleading. In Figure 7a, both objects are clearly apart and not aligned with each other. When the objects are viewed using the (orthogonal) side view, as shown in Figure 7b, the objects are again seen as being far apart from each other but at the same depth. When object $B$ is dragged along the $X$ axis and placed below object $A$, as shown in Figure 7c, object $A$ looks to be aligned with object $B$. However, when the viewing orientation is again changed, as shown in Figure 7d, it is clear that the assumption that the objects have been aligned was wrong.

These examples demonstrates that alignment of object requires modellers to navigate in the $3 \mathrm{D}$ space and view the objects using different viewports in order to validate the status of the aligned objects. Although multiple viewports are useful for providing modellers with different viewing orientation, having multiple viewports open reduces the size of the working area, as well as requiring modellers to re-orient their focus back and forth between different viewports. In fact having multiple viewports open may actually hinder task performance in some 3D environments (e.g. in CAD systems [Oh and Stuerzlinger, 2005]).

The alignment and placement problems often arise from the lack of support for spatial perception, without which recognizing the relative positions and distances between objects can be rather difficult. Furthermore, in real-world objects are placed in relation to one another, and do not float in mid-air. As such, human perception is not used to dealing with this artificial aspect of virtual 3D worlds. Although some CAD software enforce such physical rules of the real-world in terms of placement of solid objects, 


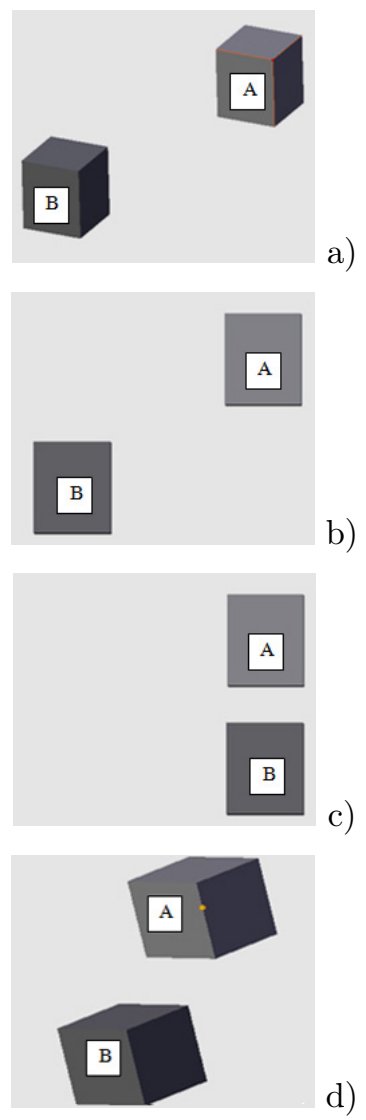

Figure 7. The process of aligning two $3 \mathrm{D}$ objects with (a) the initial view of the objects, (b) the same objects viewed from the side, (c) object A dragged to the left to be aligned with object $B$, and $(d)$ the viewing orientation is changed, showing that the objects are not aligned along the third axis.

navigation, etc. [Oh et al., 2006], this is not the case in 3D modelling software (for details see Section 5.2).

\subsubsection{Displaying objects' names}

Question 12 asked the participants to give their rating of the usefulness of displaying the names of objects in perspective mode, as provided by some $3 \mathrm{D}$ modelling software. The general idea behind displaying the names of objects is to help modellers know which objects they are working on, and enable them to identify the objects of interest accurately.

The results, as shown in Figure 2, suggest that displaying object names is not considered to be very useful by the respondents $(M=2.60, S D=0.91,1$ :not useful, 5 :very useful). The main reason given by the participants for these ratings is that displaying object names would further clutter the view of the 3D model.

\subsubsection{Occlusion, parallax effect and depth perception}

In questions 13,14 , and 15 , the participants were asked about their knowledge of the occlusion, parallax effect and depth perception, respectively. More than $90 \%$ of the participants had no understanding of the occlusion or parallax effects, while $60 \%$ of the participants did not know what depth perception was.

Although these results reflect the participants' lack of experience in 3D modelling tasks, they also highlight the fact that software applications such as Blender do not necessarily provide good support in these areas, which one would consider to be fundamental to 3D modelling.

\subsection{Summary of the findings of the pilot study}

The results of this pilot study demonstrated that all the participants had faced a range of difficulties while performing their 3D modelling assignment tasks. Even though most of these novice modellers' experiences are based on Blender,

as mentioned earlier, Blender is a typical example of polygonal 3D modelling software, and provides reasonably similar tools to other such software. Therefore, the use of Blender, instead of other 3D modelling software, cannot be the main reason for the problems encountered by our study participants.

Most of the difficulties identified in this study can be divided into one of four categories that are broadly related to focus and context awareness. These are discussed below.

\subsubsection{Maintaining position awareness}

Our study participants have highlighted that they sometimes get lost in the 3D modelling space while performing their tasks. This is evidenced from the participants' responses to Question 6, which shows that all the respondents often relied on two or more viewports while performing their tasks.

Further evidence is observed from the participants' responses to Question 7, in which they rated the importance of view all functionality as being high. This suggests that maintaining position awareness can be difficult when some of the information is not visible, which tends to happen as a result of navigation and manipulation operations. Similarly, a high difficulty rating given to Question 8 also shows that the lack of information about the eye location is problematic, which further suggests that maintaining position awareness in $3 \mathrm{D}$ space is indeed difficult.

We had assumed that providing object labels may assist users with identifying their position in 3D space. However, the participants rated this option (Question 12) as not very useful, due to it adding more visual information into an already complex environment. 


\subsubsection{Identifying and selecting objects or components of interest}

The ratings given by our study participants to questions 9 and 10 indicate that selecting a vertex or a group of vertices is considered to be difficult. Furthermore, responses to Question 6, as described above, also show that multiple viewports were often used by the participants to guide them in identifying and selecting objects of interest.

\subsubsection{Recognizing the distance between objects or components of interest}

The results of the study also showed that nearly $70 \%$ of the participants acknowledged deleting their model one or more times and starting over with new primitives. As described by many of the participants, one of the reasons for this was because they had selected a vertex (or vertices) on the wrong side of the object when reshaping their model, which had ruined the shape of their model, without them realising it at the time of reshaping, but noticing it later on when viewing the model from a different orientation, or after further modelling steps had made it impractical to use the undo command. This not only confirms the problem with selection of objects or components of interest, but also indicates that modellers find recognizing the distance between objects or components (in this case front and back vertices) difficult.

\subsubsection{Realizing the relative position of objects or components of interest}

The participants' responses to Question 11 indicated that the task of aligning objects in 3D space is not very easy. As discussed earlier, this is mainly caused by the difficulty of realizing the relative position of objects or components to each other.

\section{STUDY OF PROFESSIONAL 3D MODELLERS}

The pilot study of novice 3D modellers identified four main categories of focus and context awareness problems. Due to the broad exploratory nature of this study, and the limited experience of our participants in dealing with complex modelling tasks, a more in-depth study was subsequently conducted with professional 3D modellers. This study focused more specifically on the four categories of problems identified in the pilot study.

This new study consisted of a series of semi-structured interviews [Gorman and Clayton, 2005], during which open-ended questions [Lazar et al., 2010] were asked. The semi-structured interview method was chosen because it allowed us room for exploration, and addition of further questions to seek clarifications when required [Lazar et al.,
2010]. Beside the interviews, the participants were also observed while performing 3D modelling tasks in their workplace. Some of the interview and observation sessions were video-taped, when permission was granted to do so.

During the interviews, images of 3D models relevant to the questions were shown to the participants. The images were used for illustrating modelling situations or scenarios which may involve focus and context awareness problems as identified in the pilot study. The images used in the interviews will be presented below in related sections when discussing the findings of the study. We used a 3D model of a car for these illustrations, because most people are familiar with the structure of a car and its components (e.g. there are seats inside the car, where the engine should be, etc.). We are not suggesting that a car designer would choose a polygonal modelling software instead of a CAD system to design their car.

Furthermore, in order to better understand our participants' explanations, we also sometimes asked them to demonstrate the relevant modelling issues using their own example models. These demonstrations included when and how the difficulties would normally occur and how they were resolved. In the course of these demonstrations, the participants were observed, questions were asked for clarification, and audio or video-recordings were made when permitted.

\subsection{Interview questions}

As mentioned, the participants were interviewed using a set of open-ended questions, giving us flexibility for adding or removing questions depending on the circumstances and feedback given by the participants, which varied due to their modelling experience. The open-ended questions also allowed the participants to provide more in-depth answers to our questions, and and discuss any other issues of interest [Lazar et al., 2010]. A summary of the interview questions is given in Table A2. The questions posed in the interviews are divided into four groups:

- Modelling experience (questions 1-7): these questions probed the participants' levels of 3D modelling experiences and their software preferences.

- Methods of modelling and preparation (questions 8-15): these questions aimed to determine the participants' methods of modelling and preparation.

- Focus and context awareness problems (question 16-28): These questions were concerned with the main aim of the study. They are further divided into four categories: maintaining position awareness (questions 16-21), identifying and selecting objects or components of interest (questions 22-24), recognizing 
the distance between objects or components of interest (questions 25-26), and realizing the relative position of objects or components of interest (questions 27-29).

- Group projects and collaborative work (questions 30-38): these questions aimed to identify whether or not the four categories of problems investigated in questions 16-28 also exist in the context of group projects, and if so, how such problems occur.

Further to these questions the participants were also asked to highlight any other types of difficulties that they have previously experienced. For each difficulty that they identified, the participants were asked to explain in detail those situations that cause the problems to occur, and discuss how they overcome these problems using existing 3D modelling techniques.

\subsection{Study participants}

Open invitations were made by email to a range of companies offering 3D modelling services. No preliminary filtering of the participants was undertaken in terms of their 3D modelling software preferences. However, whenever possible, total years of working experience was taken into consideration before issuing invitations.

Out of 30 invitations sent out, 13 people working in 13 companies, spread across several different cities in New Zealand, agreed to be observed and interviewed. The participants were mainly involved in the film or computer gaming industries, and came from a broad range of typical professionals involved in 3D modelling.

Of the thirteen participants, 10 imposed a condition prior to the interviews that video-taping the sessions or viewing of the $3 \mathrm{D}$ models that they were working on was not allowed due to confidentiality or copyright issues. However, all the participants agreed to have their interviews audio-taped.

\subsection{Data collection and analysis}

The main method of data collection during the interviews and observations was through audio-recording (and some video-recording when allowed), complemented with some hand-written notes. The length of the audio recordings varied from 30 to 50 minutes, depending on the actual interviews and the time taken to demonstrate modelling tasks. The total duration of the sessions ranged from one to two hours.

Analysis of the recorded data involved listening to the audio recordings, watching the recorded videos, transcribing the conversations, taking notes of any important observations, and looking at the hand-written notes. This was an iterative process, requiring several passes through the recorded and transcribed data, according to the thematic analysis method, originally proposed by [Dey, 1993], and widely used since for analyzing qualitative data collected through interviews and observations [Braun and Clarke, 2006].

The results of this analysis are presented below, based on the categorization of the of the open-ended questions used in the interviews (see Section 4.1).

\subsection{Modelling experience and methods used}

Table A3 presents a summary of the participants' 3D modelling experiences and their use of modelling software. Most of the participants $(85 \%)$ had five years or more of 3D modelling experience. All 13 had experience of using MAYA, 9 with 3ds Max, 5 with ZBrush, 3 with Blender, 1 with Lightwave and 1 with Softimage. Even though the interfaces of these software are different, their functionalities are generally similar (except for ZBrush to some extent). Hence the differences, advantages and disadvantages of the individual 3D modelling software are not considered here in the analysis of our study results.

The participants were asked to describe the most challenging project they had worked on (Question 3). The responses indicated that $3 \mathrm{D}$ modelling tasks can be divided into two categories. The first category is referred to as a "well planned and structured 3D modelling" task, where the expected finished 3D model is well-defined. The second category is an "ad hoc type of modelling" task, where the target output is not well-defined. Majority of the participants (9 of 13) noted that working on the second type of modelling tasks is often the most challenging type of project.

The participants were also asked to estimate the average time they take to complete their 3D modelling tasks (Question 4). The aim of this question was to estimate the complexity of the projects the study participants work on. Of the 13 participants, 8 reported that they have been involved in 3D modelling tasks that have taken between one to three months to complete. This indicates that our participants had some experience of working on large complex projects.

In terms of the range of their experiences, the participants were asked whether they do computer animation as well as 3D modelling (Question 5). Nine of the participants worked on computer animations as well. In response to the related Question 6, seven of these 9 participants said they spend more than $50 \%$ of their time on modelling, while the other 2 participants spend more than $50 \%$ of their time on animations. Finally, in response to Question 7, six of the participants ranked the modelling tasks as being more difficult than animations, while 3 ranked animations as being more difficult. 
In terms of the methods of modelling and preparation, the participants were asked whether they recycle existing 3D models (Question 8). Eight (62\%) of the participants responded that they preferred to create $3 \mathrm{D}$ models from scratch instead of recycling existing models. However, the participants said that when possible, using existing models could reduce their work by as much as $50 \%$.

The participants were also asked whether they had ever worked on, or improved, someone else's model (Question 9 ). Eight of the participants noted that they had used someone else's model. Of these eight, four described recycling someone else's model as being both easy and practical, while the other four claimed that making further enhancement to someone else's model was a difficult task. These four participants, not in favour of modifying someone else's model, claimed that they normally did not have enough information (e.g. the overall structure of the model) when doing this type of tasks.

In response to Question 11, most of the participants (12) preferred to do their modelling when they have everything clear in their mind. The same number of participants (12) also preferred to have other 3D modelling elements such as lights, textures, and colors well defined before they start their modelling tasks (Question 12). In a related question (Question 13), eight (62\%) of the participants said that they generally draw the textures from scratch rather using existing textures.

When asked about the type of viewports they most commonly use (Question 14), 8 of the participants said they preferred to use an orthographic view, 3 preferred a perspective view, and the other two noted using both types of views. Similar responses were obtained from the participants when they were asked to describe their viewing preference when editing objects (Question 15). In the edit mode, 9 of the participants preferred to work in orthographic view, 2 preferred perspective view, and the other 2 preferred to use a combination of both views.

In summary, responses to questions 1-15 demonstrate that our study participants were very experienced, and had extensive working knowledge of various modelling methods.

\subsection{Focus and context awareness problems}

At this point it is important to note that before interviewing the study participants about the kinds of focus and context awareness problem they may have faced in their modelling tasks, the participants were given a detailed explanation of what is meant by focus and context awareness. This was necessary because the participants were generally unaware of the relationship between focus and context awareness and 3D modelling tasks. Based on these detailed explanations and examples given to them, the participants were able to relate their modelling experiences to the four categories of focus and context awareness problems that this study aimed to investigate.

As mentioned earlier, the participants were also asked to describe any problems other than those related to focus and context awareness. Most of these other problems identified were however generally minor and are outside the scope of this article.

Table A4 provides a summary of the participants' responses indicating whether or not they had faced focus and context awareness difficulties while performing 3D modelling tasks. Detailed explanations of the issues related to these categories are provided in the following sections.

\subsubsection{Maintaining position awareness}

Regarding the issue of maintaining position awareness, the participants were asked (Question 16) to discuss how they know what objects they are looking at, and from which angle they are being viewed. Eleven (84\%) of the participants said that they relied on extra viewports to display multiple viewing angles of objects to guide them during the navigation process. The other two participants, however, relied on reference drawings or sketches of objects instead of opening extra viewports. These two pointed out that opening extra viewports reduced the size of their screen workspace.

Question 17 asked the participants whether they have experienced any difficulties recognizing the orientation of objects in 3D scenes. Once again, 11 of the participants acknowledged that they often fail to recognize the orientation of objects, particularly in wireframe mode. When asked in Question 18 to describe solutions to this particular problem when it occurs, the participants said that they often rely on one of three different solutions to this problem: look at the object in other viewports or printed reference material, zoom out of the scene being viewed (e.g. using view all), or change the mode of the object being displayed (e.g. from wireframe to solid mode).

A very similar pattern is also observed during the manipulation process. In questions 19-21, the participants were asked about the approaches they take during the manipulation process, and whether they have encountered any problems realizing the extent of their manipulation, or recognizing objects' position and orientation as the result of manipulation. In response to Question 19, eleven (84\%) of the participants stated that they rely on extra viewports to guide the manipulation process. For questions 20 and 21 , the same number of participants claimed that they had sometimes experienced problems understanding what they had done during the manipulation process, as well as not recognizing objects' position and orientation.

Figure 8 illustrates the difficulty that modellers may have faced when navigating in a 3D space. In this example, 
a 3D car model (Figure 8a) is shown in wireframe mode. From this viewing angle, the viewer should be able to identify the orientation of the displayed model. However, when navigating, the model may be seen from a different orientation as illustrated in Figure $8 \mathrm{~b}$. In this case, due to the overlapping edges, it would be difficult to recognize the orientation of the car. In Figure $8 c$ the display mode is changed from wireframe to solid, thereby, rectifying this confusion and making it clear that the model in Figure 8b is being viewed from underneath. Note that this problem is made worse by the use of orthographic mode, and would be less of a problem in perspective mode.

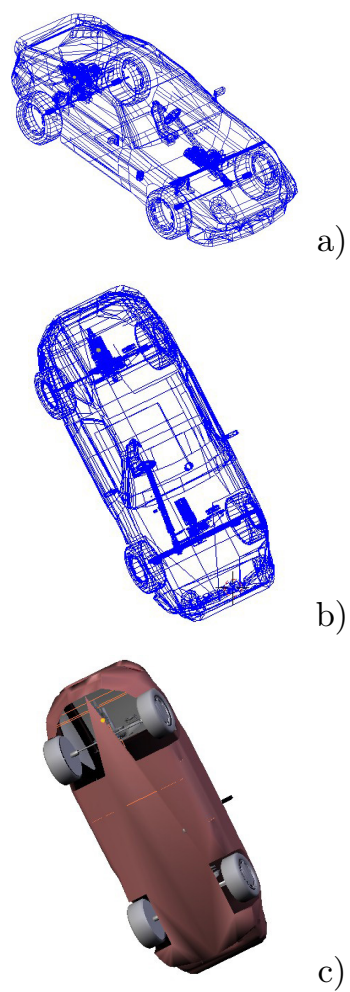

Figure 8. An example of maintaining position awareness problem caused by navigation.

A common solution to maintaining position awareness, as pointed out by a few of the participants, is to open a second viewport in which the model is continuously shown in solid mode. Figure 9 illustrates the benefit of using multiple viewports. Figure 9a shows the orientation of the model before navigation, while Figure 9b shows its orientation after some navigation. Although the differences between these two orientations are not so noticeable in the wireframe views, the solid mode (shown on the right for each case) provides a better perspective of the model's orientation. Unfortunately however, in most conventional 3D modelling software there is no link between multiple viewports. This means that the orientation of the model being viewed in two viewports will not remain the same when navigation is performed in one of the viewports. One of the ways of achieving the linked display effect in some software, as shown in Figure 9, is by splitting a single viewport into two.
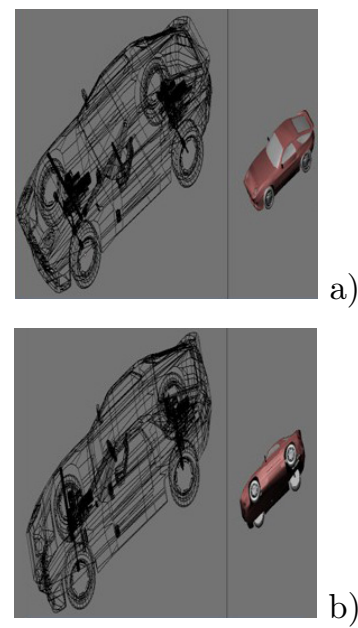

Figure 9. Orientation of the model (a) prior to navigation, and (b) after navigation, in both wireframe and solid modes.

It should also be noted that in some cases displaying the model in both solid and wireframe modes may not solve the problem of focus and context awareness during the navigation and manipulation process. For instance, the problem would persist when the selected objects or components of the model are internal and cannot be seen in the solid mode (see Figure 10).
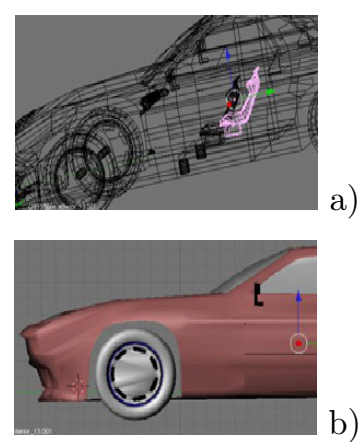

Figure 10. The selected front seat is (a) visible in wireframe mode, and (b) not visible in solid mode.

\subsubsection{Identifying and selecting objects or components of interest}

The participants were asked to describe how they manage selection of objects and components of 3D models (Question 22), how they make sure that they are selecting 
the correct one (Question 23), and whether they have encountered any problems when performing this type of tasks (Question 24). In response to these three related questions, ten $(77 \%)$ of the participants mentioned that they have encountered problems when performing such operations, and described various methods for managing the object or component selection process. These methods include selecting and hiding the unwanted objects, putting objects in different layers and not displaying the unwanted layers, performing navigation moves around objects, zooming in and out, opening multiple viewports with different views, and generally relying on trial and error to determine the status of the selected objects or components.

To investigate this issue further, the participants were asked to look at a model in wireframe mode (as shown in Figure 11a) and to identify the location of the selected tyre (i.e. rear left). All the participants were able to identify the location of the selected tyre correctly without the aid of other tools such as using a second viewport. The participants were then presented with a rotated image of the same model (as shown in Figure 11b), and asked to identify whether the selected tyre was the rear left or right tyre. More than $80 \%$ of the participants gave the wrong answer to this question by saying that it was the rear right tyre. However, when the model was shown in solid mode (Figure 11c), the participants were able to correct their mistake. This demonstrates that having multiple viewports and displaying a model in different modes are needed to help even experienced modellers to recognize the orientation of the model after a manipulation process.

Unfortunately this is not always sufficient though, as demonstrated by another example, in which the entire car is rotated even further, as shown in Figure 12. When the participants were shown this image with both solid and wireframe modes, and asked to identify the location of the selected tyre, they found it difficult to answer even with the support of the solid model. In fact, more than $80 \%$ of the participants gave the wrong, by assuming that the selected tyre was the rear right tyre instead of the rear left. This confusion is caused by the fact that the selected object is highlighted and looks closer to the viewer. The participants were also rather confused when comparing the wireframe and solid views of the model which seemed to contradict each other.

One could of course argue that the problem demonstrated in this example is caused by the fact that the implementation of highlighting method in some software displays highlighted objects closer to the viewer, and other better highlighting solutions could be found. However, due to a lack of studies such as ours, these problems have not been identified previously, or addressed by most existing 3D modelling software.
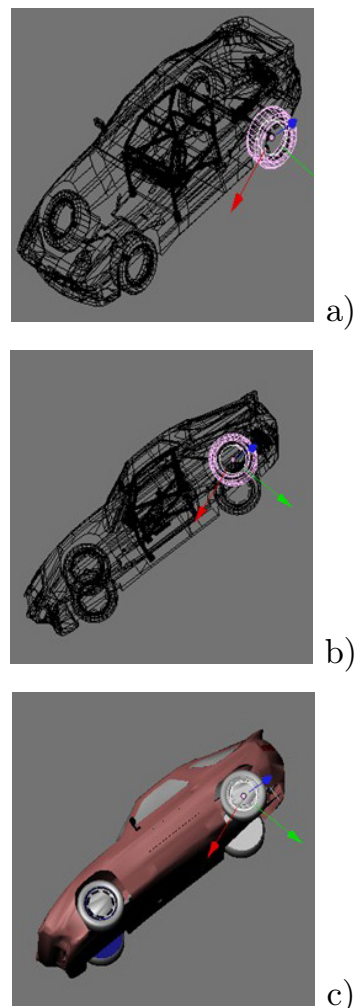

Figure 11. View of a model with the rear left tyre selected in (a) initial wireframe mode, and after rotation in (b) wireframe mode, and (c) solid mode.
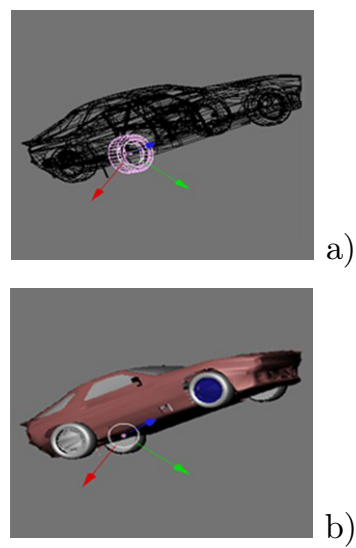

Figure 12. Model of the car after further rotation in (a) wireframe mode, and (b) solid mode.

\subsubsection{Recognizing the distance between objects or components of interest}

The participants were asked whether they have had any difficulties recognizing the distance between components or objects of a model (Question 25), and how they would verify which components are nearer to them (Question 26). Twelve (92\%) of the participants admitted that they sometimes find it difficult to recognize the distance 
between components or objects (i.e. how far apart they are) or recognize their depth order. Due to the depth order problem in particular, they often select the wrong object or component, and this causes them to either repeat or perform unnecessary steps to correct their mistakes, which should be avoidable from the outset. The participants also pointed out that they rely on various techniques to assist them with solving their mistakes. These techniques include zooming, moving around objects (navigation), opening multiple orthographic viewports, changing the model editing mode from wireframe to solid, and using back surface removal. The back surface removal technique (as shown in Figure 13) is effective for reducing the amount of information being displayed, but it removes the components of the far side of the selected object, and consequently, the relationship between near and far components is lost.
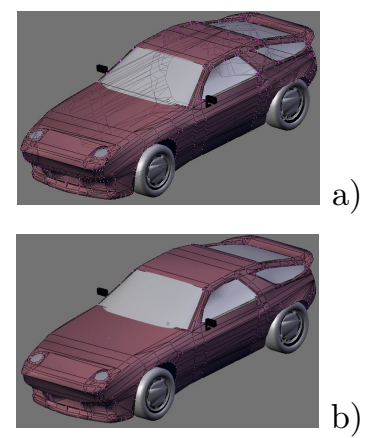

Figure 13. Model of a car (a) without back surface removal all the vertices and edges of the selected object (car body) are shown, and (b) with back surface removal only the front vertices and edges are shown.

\subsubsection{Realizing the relative position of objects or components of interest}

Question 27 asked the participants whether they prefer to create a model as a single object, or as a series of individual objects modelled separately and then combined together. The aim of this question was to identify any problems encountered during the process of assembling objects. Almost all the participants (12) said that they prefer to create individual objects separately and then assemble them together. However, in response to Question 28 , eight of these 12 participants said that they found the process of assembling different objects together to be a difficult one, in which they encounter problems such as selection, alignment, etc. as described earlier.

In Question 29, the participants were asked to describe the methods they use to reduce the difficulty of recognizing objects' relative position when assembling them. Their responses showed that more than $90 \%$ of them rely on multiple viewports in one way or another to ease their tasks when assembling objects.

\subsubsection{Group project or collaborative work}

The final part of the interviews investigated the participants' experiences relating to group projects and collaborative types of work (questions 30-38). The purpose of these questions was to find out any focus and context awareness problems which may occur specifically in these types of collaborative work, that may not relate to performing modelling tasks by a single modeller. Therefore, in this section we only present issues relevant to focus and context awareness.

In response to Question 33, all the participants who had experienced group work noted that there is often no single individual who decides on the scale or textures of the model. Any proposals or ideas are generally discussed and agreed upon by the team members.

These participants mentioned that usually an individual team member is assigned the task of the final arrangement of objects in a model or scene (Question 35), and that this particular individual usually has no right to modify or amend objects created by others (Question 36). The task of improving the individual objects is undertaken by the owner or creator of the object. The aim of this question was to understand the difficulties (if any) that are faced by an individual when combining or modifying models created by others.

Furthermore, in the related Question 37, the participants stated that the individual responsible for assembling the final scene performs the task of placing the 3D objects at their designated locations in the $3 \mathrm{D}$ space. While performing this task, the individual involved often faces problems caused by the quality of the $3 \mathrm{D}$ objects failing to meet the scale or standard of the project.

Finally, in response to Question 38, the participants noted that they often experience some difficulties in placing individual objects in a $3 \mathrm{D}$ space due to the problem of not recognizing the relative position of objects (a point which has been discussed earlier).

\subsection{Summary of the findings}

The results of the study of professional 3D modellers have further highlighted and clarified issues related to the four categories of problems associated with maintaining focus and context awareness in 3D modelling tasks, as identified in the earlier pilot study of novice modellers. These issues are summarized as follows:

- Maintaining position awareness: This category of problems refers to modellers' difficulty in maintaining awareness of the position and orientation of objects of a model when performing manipulation and 
navigation tasks. Findings from the study show that modellers often rely on techniques such as opening multiple viewports, hiding, layering, and changing model display mode to solid or wireframe when performing these tasks. These techniques enable modellers to maintain or re-establish their understanding of context and recognize what they are looking at in terms of their focus. However, none of these techniques is able to fully support maintaining position awareness. For instance when using multiple viewports, the area of focus and context are separated, which requires modellers to switch their attention from one viewport to another. In addition to this problem of discontinuity, the objects being viewed in each viewport are often displayed in different zoom level or from different viewing orientation. These two problems occur because in most 3D modelling software viewports are treated independently of each other. Furthermore, using multiple viewports also divides screen realestate into a series of smaller sections which reduces the amount of information that can be displayed in each of them and condenses the size of the graphical data (i.e. model) on the screen. Consequently, modelling tasks tend to become more complicated because the objects and components of the model overlap each other even more.

- Identifying and selecting object or components of interest: This category of problems refers to situations where modellers face some difficulties in selecting objects or components of interest correctly. As discussed earlier, overlapping of objects and components is a factor that causes this type of problem to occur. In a cluttered 3D model viewing situation, modellers have to rely on different techniques such as hiding and layering to overcome this problem. These techniques reduce the amount of clutter and therefore increase the accuracy of selecting objects or components of interest. However, they also tend to eliminate modellers' ability to maintain their awareness of the relationship between the objects or components of interest with the others (i.e. context). As a result, modelling tasks which are constrained by these hidden objects can become difficult.

- Recognizing the distance between objects or components of interest: This third category of problem relates to recognizing the location of objects or components in a virtual $3 \mathrm{D}$ modelling space. It includes the difficulty of recognizing the near and far objects or components. This type of problem often takes place when models are displayed in the wireframe mode. As discussed earlier, modellers often use multiple viewports to overcome this problem by viewing objects or components of interest from different angles or orientations. This reliance on multiple viewports, however, leads to break down of focus and context awareness as discussed before.

- Realizing the relative position of objects or components of interest: This category of problem is related to the difficulty of placing or aligning objects in a virtual 3D space. Findings of the study, as discussed earlier, indicate that modellers often have difficulties in performing this type of task using conventional 3D modelling software. The study showed that modellers usually need to view the model being manipulated from various angles (e.g. top, side, and front) in multiple viewports. This, once again, leads to problems associated with relying on multiple viewports.

\section{SUPPORTING FOCUS AND CONTEXT AWARENESS}

Potential solutions for better supporting focus and context awareness in 3D modelling tasks may be found in two different (and somewhat independent) fields of existing research, namely those relating to focus and context awareness, and those dealing with 3D environments. The literature on focus and context awareness generally deal with providing more effective visualizations of focus and contextual information, while those related to $3 \mathrm{D}$ environments largely aim to support better user interaction with virtual 3D worlds. As such, here we divide our presentation of potential solutions to focus and context awareness problems into visualization and interaction techniques.

\subsection{Visualization}

Most of the problems that our studies have identified can be addressed by providing more effective visualizations of focus and context. Although research dealing specifically with focus and context awareness in 3D environments is not extensive, there is a large body of work dealing with issues related to visualization of focus and context information in 2D environments. Cockburn et al. [2008] provide a review of focus and context in 2D interfaces. They divide their review into four categories: overview + detail (spatial separation), zooming (temporal separation), focus + context (seamless focus in context), and cue-based techniques.

We use this categorization here to discuss the relevance of some of the existing $2 \mathrm{D}$ focus and context awareness visualization techniques as potential solutions for addressing the problems our studies have identified in relation to $3 \mathrm{D}$ modelling tasks. 


\subsubsection{Overview+detail}

These types of visualizations present an overview of the entire information space along with a detailed view of part of the information space in separate spatial areas (e.g. windows) [Hornbæk et al., 2002]. Overview+detail techniques are commonly used, for instance, in map visualizations and computer applications (e.g. thumbnail document viewers). Although these techniques improve user navigation, due to the spatial separation of the overview and detail spaces, their use can require more mental effort, and they can be slower to use than other visualizations relying on a single view [Hornbæk et al., 2002].

Overview + detail is certainly supported by almost all 3D modelling software that allow multiple viewports, in which users can choose to have different combinations of overview (zoomed-out) and detail (zoomed-in) views of their 3D modelling workspace. As our pilot study shows, multiple viewports are commonly used, both in orthographic and perspective modes, to simultaneously view models from different positions (Section 3.3.3), particularly when placing and aligning objects (Section 3.3.7). Similarly, our study of professional modellers showed the use of multiple viewports to deal with maintaining position awareness during navigation and manipulation tasks(Section 4.5.1), when trying to recognizing the distance between objects or components of interest (Section 4.5.3), as well as when attempting to realize the relative position of objects or components of interest (Section 4.5.4). However, our studies also show that such combinations of viewports on their own are not always sufficient in providing modellers with focus and context awareness needed to carry out their tasks (Section 4.6).

Furthermore, Plaisant et al. [1995] specify that for overview +detail techniques to be effective they must provide a tight coupling between the two views, so that navigation or selection actions in one are immediately reflected in the other [Ahlberg and Shneiderman, 1994].

Although it is possible to lock viewports together in some $3 \mathrm{D}$ modelling software, this is not automatic, and as such navigation tasks in one viewport may not necessarily change the views of other viewports. This makes maintaining position awareness challenging even with the use of multiple viewports as identified in our studies (Section 4.5.1). Most 3D modelling software also do not enforce a size ratio factor between the areas of overview and detail viewports. Although the area size factor, along with the zoom factor, are task dependant, there are recommended area size factors (e.g. less than 25 [Plaisant et al., 1995]) which should be followed.

In most 3D modelling software the size of viewports can be modified independently of each other, or they are forced to be the same size. In addition, as more viewports are opened, with the limited screen real-estate available, the working area in each viewport becomes even smaller, causing even more overlapping of objects and components of the model (Section 4.6).

\subsubsection{Zooming}

Zoom-based visualization techniques have existed for over two decades. Perlin and Fox [1993] introduced the concept of portals, to allow users in a collaborative setting to roam and view different parts of an "infinite shared desktop" using magnifying glasses (i.e. magnificationbased zooming). They also introduced the concept of semantic zooming, which relates to the amount of semantic detail presented at different zoom levels.

All 3D modelling software provide magnification-based zooming. Semantic zooming is also supported, to some extent, by allowing the viewing of models in different modes (e.g. solid view and wireframe), as well as in the level of detail shown at different zoom levels. Also, in cases where objects are grouped and named, details such as object names are shown when sufficient zoomingin is applied. Although as our pilot study participants pointed out, such extra detail can actually make modelling tasks more challenging by further cluttering the visual information space (Section 3.3.8).

Hornbæk et al. [2002] point out that zoomable interfaces generally combine zooming (change of scale) with panning (change of visible area). They also discuss how in most cases zooming and panning are changed linearly through direct user input (e.g. via a mouse). Non-linear zooming and panning, on the other hand, can be in one of three forms: goal-directed (direct zoom to a particular scale), combined zooming and panning (extensive panning leading to automatic zooming), and automatic zoom to an object of interest [Hornbæk et al., 2002].

An example of this type of automatic zoom is through the use of view all function in Blender, which was considered to be very useful by the participants of our pilot study (Section 3.3.4). This can, however, lead to a drastic change of the viewing position and size, which can in turn cause difficulties in maintaining position awareness (Section 4.5.1).

Although various studies that have investigated the effectiveness of zooming, with or without overview, in $2 \mathrm{D}$ visualizations have been inconclusive, there is some evidence for benefits they offer [Hornbæk et al., 2002].

Similarly, our studies show that although zooming, in combination with multiple viewports, is commonly used in 3D modelling tasks for aligning and placement of objects (Section 3.3.7), maintaining position awareness (Section 4.5.1), identifying and selecting objects and components (Section 4.5.2), and recognizing the distance between objects or components of interest (Section 4.5.3), 
zooming methods are not always satisfactory in overcoming focus and context awareness problems associated with these tasks (Section 4.6).

\subsubsection{Focus+context}

Unlike the previous two types of techniques, which either separate focus and context views spatially (overview + detail) or temporally (zooming), focus + context techniques combine focus and context views into a single visualization, and therefore, reduce reliance on short-term memory required for assimilating views separated over space or time [Cockburn et al., 2008].

As discussed in the previous two sections, our studies have identified that the use of multiple viewports (which causes spatial separation), with or without zooming (which causes temporal separation), tend to be insufficient in supporting effective focus and context awareness in 3D modelling tasks. Focus + context techniques, on the other hand, aim to reduce spatial and temporal separation, and therefore, may offer better support for focus and context awareness.

Many focus + context techniques tend to be distortionbased, and use differential scaling as the visualization moves away from the focus of interest towards context. These methods include bifocal display [Spence and Apperley, 1982], fisheye view [Furnas, 1986, 2006], perspective wall [Mackinlay et al., 1991], and others (for a review see [Leung and Apperley, 1994]). Although in some cases distortion-based techniques such as fisheye view perform better than, for instance, zoom-based techniques [Schaffer et al., 1996], they distort viewing space, and therefore, suffer from misrepresentation of underlying data, and pose challenges to target acquisition [Cockburn et al., 2008].

As such, distortion-based techniques are not generally suitable for 3D modelling tasks where presentation of spatial relationships is important, for instance, for recognizing the distance between objects or components of interest (Section 4.5.3), or realizing their relative positions (Section 4.5.4). Similarly, in 3D modelling, the ability to find and select targets (i.e. objects or components of interest) is critically important for task performance (Section 4.5.2), making distortion-based techniques less than ideal for such tasks.

There is another category of non-distortion-based focus + context techniques, which utilize a number of opaque and transparent or semi-transparent (using alphablending [Porter and Duff, 1984]) layers to combine focus and contextual views of information spaces into a single view. One of the earliest examples of such a multi-layer visualization using a transparent layer is Magic Lens [Bier et al., 1993], which not only provides magnification type zooming of focus area over the context, but also semantic zooming of the level or type of information presented in the focus area. Other similar techniques are demonstrated by Lieberman [1994], and Cox et al. [1998]. Harrison et al. [1995] conducted experimental evaluation of transparency levels, as well as other visual design elements, that impact usability of multi-layer visualizations. They suggest a transparency level of $50 \%$ as a good trade-off for supporting tasks on both the foreground and background layers.

Baudisch and Gutwin [2004] have, however, identified a major problem with multi-layer techniques using alphablending, in that they introduce visual ambiguity. This makes it difficult for viewers to identify the actual layer on which different visual information are presented (also noted by [Cox et al., 1998]).

As many 3D modelling tasks require accurate perception of depth-ordering (Section 4.5.3), alpha-blended multi-layer techniques are not likely to be useful for these tasks.

To avoid visual ambiguity of alpha-blending techniques, other methods have been proposed which tend to visually separate focus from context, while presenting both in a single view. An example of these is the cutaway technique, used, for instance, in volumetric medical data visualizations [Viola et al., 2005]. Various interactive cutaway techniques have been implemented to allow cutting based on a location specified by the user [Bruyns et al., 2002], automatically around the object of interest [Diepstraten et al., 2003], or based on the hierarchy of the objects of a model [Li et al., 2007]. The cutaway technique removes parts of the rendered $3 \mathrm{D}$ objects, leading to the lack of contextual information [Moura Pinto and Dal Sasso Freitas, 2011]. To solve this problem, at least in the case of rendered volumetric data, other techniques have been developed. These include ClearView [Krüger et al., 2006] which relies on the use of explicit ordered layers, hybrid visibility [Bruckner et al., 2010] used for generating 3D illustrations using 2D and 3D layers, importance-aware composition [Moura Pinto and Dal Sasso Freitas, 2011] which also requires manual layering based on importance, deformation based techniques [McGuffin et al., 2003] which distort the context in which the area of focus is shown, and dynamic transparency [Elmqvist et al., 2007] that supports viewing of occluded 3D objects by changing the transparency of occluding surfaces (for a more complete review of the volumetric techniques see [Krüger et al., 2006] and [Bruckner et al., 2010]).

All these techniques are however rendering techniques, applied at the pixel or voxel level to render several layers into a single composited rendered view.

Therefore, these techniques have not been used in 3D modelling situations, where the user needs to interact with modelling components and objects (e.g. select a vertex or a group of vertices), rather than a series of pixels or voxels 
which are rendered and presented as a single image. A more interesting cutaway technique has been developed by Knödel et al. [2009] for 3D polygonal models (rather than volumetric data as above), which although is currently proposed for illustration rendering purposes, should be investigate for 3D modelling tasks.

There are also a number of hardware-based focus + context techniques, which aim to address visual ambiguity or distortion-related problems caused by software-based focus+context visualizations. For example, the mixed-resolution displays setup [Baudisch et al., 2002] uses a small high-resolution display for focus and a larger projected lower-resolution display for context. These type of systems have however become less useful with more widespread availability of affordable large high-resolution displays.

Other hardware-based solutions include the use of multi-layered displays [PureDepth, 2014] in 2D visualizations, where the physical front layer of the display is used for focus and the rear layer used for context [Wong et al., 2005, Masoodian et al., 2004]; and augmented reality techniques for combining context and focus [Zollmann et al., 2012].

Finally, with the more wide-spread availability of 3D stereoscopic displays in recent years, there is growing research in designing for such displays, particularly in computer games [Schild et al., 2013], which have shown some positive benefits in terms of gameplay task performance [Kulshreshth et al., 2012]. Interestingly new extensions to 3ds Max [Autodesk, 2014] are planned to provide stereo camera design tools for $3 \mathrm{D}$ modellers in the near future.

\subsubsection{Cue-based}

Unlike the other three categories of focus and context visualizations, which define focus based on an area of interest (i.e. a sub-space of the context), cue-based techniques define focus across the context based on some specific properties [Cockburn et al., 2008]. Objects of focus which satisfy a particular criteria are displayed in a manner that separates them from context. Examples of such techniques include the semantic depth of field [Kosara et al., 2001, 2002] method for rendering 2D and 3D images, in which objects of interest (focus) are sharply displayed while the less relevant areas (context) are blurred. However, as with volumetric rendering techniques described above, the result of this technique is a rendered image.

Most 3D modelling software allow users to define 3D objects (i.e. components grouped together) which can then be selected easily, for instance by using the names assigned to different grouped objects (Section 3.3.8). This grouping technique is usually used for selecting an object and hiding the remaining parts of the model, particularly when trying to identify and select objects or components of interest (Section 4.5.2). However, as mentioned earlier, in such cases the context is hidden from the view, causing awareness problems for the user (Section 4.6).

\subsection{Interaction}

In their recent comprehensive survey of interaction techniques for 3D environments, Jankowski and Hachet [2013] use the categorization of earlier surveys ([Hand, 1997, Bowman et al., 2001]) to divide 3D interaction into: navigation, selection and manipulation, and system control. We also found this categorization useful for our discussion here.

\subsubsection{Navigation}

Navigation refers to the process of moving around in a virtual 3D space. Navigation can be subdivided into the motor aspect of navigation, or what Bowman et al. [2001] refer to as travel, and the cognitive aspect, referred to as wayfinding (see below).

Mackinlay et al. [1990] identify at least four types of movement (or travel) in interactive 3D environments: (1) general exploratory movement, (2) targeted movement with respect to a specific target, (3) specified coordinate movement to a precise position and orientation, and (4) specified trajectory movement along a position and orientation trajectory.

General and targeted navigations are perhaps the most common in 3D modelling tasks. Specified coordinate movements, although possible, are not as frequent, while specified trajectory movements are not generally done in $3 \mathrm{D}$ modelling while creating the models themselves. Although navigation is clearly important in 3D modelling tasks, as our studies have shown, commonly used techniques as such targeted navigation are not always sufficient for maintaining position awareness, and can lead to problems related to not knowing the viewing position or orientation even after simple navigation activities (Section 4.5.1).

Unfortunately, most techniques proposed for supporting better navigation in 3D environments (see [Jankowski and Hachet, 2013] for a review) are more suitable either for users' interaction with CAD systems or navigation in already-made (i.e. not in editing mode) virtual 3D worlds (e.g. virtual tours, 3D computer games, etc.). The underlying aim of all these techniques is to allow users' navigation automatically, or semi-automatically, through an existing 3D space by controlling or limiting user's degree of freedom in moving their viewpoint. Examples of such techniques include Point of Interest Logarithmic Flight [Mackinlay et al., 1990], Path Drawing for walkthrough [Igarashi et al., 1998], Speed-couple Flying with Orbiting [Tan et al., 2001], HoverCam [Khan et al., 
2005], and a range of others based on camera control mechanisms (for a review see [Christie and Olivier, 2009]).

Besides removing some of the control from the viewer, the main reason for why these techniques are not very useful for 3D modelling scenarios is because they assume certain properties or assign some constraints to objects presented in the virtual 3D world, often mapping those of real physical world (e.g. solid objects such as walls cannot be penetrated, movements are done along existing paths, etc.). Such assumptions are however not valid or useful in 3D modelling tasks, when the modellers may need to manipulate and modify objects internally, particularly in wireframe mode which is commonly used for vertex selection and manipulation type tasks (Section 3.3.6).

The problem of maintaining position awareness, as identified in our studies (Section 4.5.1), is less about the travel or movements aspects of navigation and has more to do with wayfinding. Wayfinding [Darken and Sibert, 1996], is related to how users build up a mental model of the virtual 3D world [Jankowski and Hachet, 2013]. In wayfinding, the actual navigation is not the goal of interaction, but only the means of accomplishing a specific task [Darken and Sibert, 1996]. Ease of navigation (travel), however, clearly contributes to improved wayfinding [Elmqvist et al., 2008].

Despite its importance to position awareness in 3D modelling tasks, wayfinding has not been investigated in this context specifically (i.e. in modelling tasks). Most proposed wayfinding techniques tend to suggest solutions for improving interaction of users (not modellers) with virtual 3D worlds by acquiring and using their spatial knowledge of the environment [Bowman et al., 2001]. These include the use of landmarks [Vinson, 1999], personal marks [Grammenos et al., 2006], as well as the use of semi-transparency (Section 5.1) for showing the internal parts of occluded surfaces [Knödel et al., 2009].

One of the useful techniques developed for CAD systems, which could help with position awareness in 3D modelling environments, is the ViewCube [Khan et al., 2008]. ViewCube was developed as an orientation indicator to show the current view orientation of the user, as well as allowing change of orientation when needed. ViewCube is one of several proposed Safe 3D Navigation solutions for by Fitzmaurice et al. [2008]. The other potentially useful Safe 3D Navigation tool is the green-ball pivot point indicator for navigation operations (e.g. rotations).

Participants of our pilot study found the lack of viewer's eye location indicator in Blender to be problematic (Section 3.3.5) for maintaining position (e.g. orientation) awareness. Tools such as ViewCube would resolve such problems.

\subsubsection{Selection and manipulation}

Most research investigating selection methods deal with selection of objects in interactive (and often immersive) virtual 3D environments and games,

while issues related to selection of objects or components in 3D modelling tasks, which our studies have shown to be challenging (Section 4.5.2), have received little attention.

Research on objection selection have investigated techniques that range from conventional ray-casting (which perform badly on small or distant targets [Poupyrev et al., 1996]) to its modified versions [Argelaguet and Andujar, 2009], to more novel techniques such as semantic pointing [Elmqvist and Fekete, 2008] which shrinks empty space to enlarge potential selection targets. Experience from 2D environments, however, indicate that distortion-based techniques seem to actually make target selection more difficult [Gutwin, 2002].

In general, many of these 3D selection techniques are similar to those found in 2D applications (for a review see [Balakrishnan, 2004]) and do not scale well to cases such as 3D modelling where a large number of targets (e.g. vertices, edges) are often located in close proximity (Section 3.3.6). One potential solution to selection of small targets in 3D space is the 3D volume cursor proposed by Zhai et al. [1994], which has unfortunately not been investigated further.

There are also a number of object selection methods that aim to overcome the problem of occlusion. Most of these deal with 2D user interface environments, which can become overcrowded with many open windows. Ramos et al. [2006] provide a review of these techniques, and present two of their own, which either present occluded objects in a 3D stacked layers (Thumbler), or in a spatially separated collection of thumbnails (Splatter). A user study of these techniques, comparing them with conventional layers palette, found several advantages and shortcoming, and also led to a third technique being proposed (Depth Wall). Although these methods mainly deal with 2D settings, they can be investigated in 3D modelling tasks for showing depth ordering of objects.

However, it is unlikely that they will be useful for showing small objects, a large number of objects, or small components which lack visual differences. These tend to be exactly the types of situations encountered in 3D modelling tasks, when users often need to select a vertex or a group of vertices (Section 3.3.6).

Manipulation tasks at the object level (e.g. scaling, rotation, etc.) are generally well supported by most 3D modelling software, as well as CAD systems. At the component level (e.g. vertices, edges), most of the challenges faced by 3D modellers are related to component selection and not necessarily component manipulation. As 
such, component or object manipulation issues are not considered here (for a review of $3 \mathrm{D}$ object manipulation techniques see [Jankowski and Hachet, 2013]).

\subsubsection{System control}

System or application control tasks refer to selection of commands provided by software applications, to either change the state of the system or its mode of interaction [Bowman et al., 2001, 2004]. Most 3D modelling software, including those used by our study participants, generally use system control techniques developed for 2D applications, and present their user interface components (e.g. menus, toolbars, buttons, dialog boxes, etc.) in 2D.

As mentioned earlier (Section 1, although 3D modelling software offer a large number of system controls, these can generally be learnt over time with practice, and therefore were not investigated specifically in our studies. Interested reader may, however, find the review of system control techniques by Jankowski and Hachet [2013], and a number of novel techniques by Fitzmaurice et al. [2008] useful.

\section{CONCLUSIONS}

This article has presented two complementary studies of problems faced by 3D modellers using conventional modelling software to perform their tasks. The first of these was a questionnaire-based study, which took an exploratory approach to investigate the broad range of problems novice 3D modellers encounter, the situations that lead to such problems, and the ways in which modellers attempt to overcome them using existing software tools.

The findings of this study led to the formulation of four specific categories of focus and context awareness problems, which were then investigated in a more comprehensive second study, involving interviews and observation of professional 3D modellers.

The results of the second study confirmed our categorization of the four types of focus and context awareness problems faced by $3 \mathrm{D}$ modellers, and shed more light on the nature of these problems in terms of when and how they occur, and what remedial steps are taken by modellers given the limited range of tools and options provided by modelling software. This study also showed that although the professional modellers participating in it were much more experienced than novice modellers of the first study, their experience of working for many years had not really enabled them to overcome these fundamental focus and context awareness problems. Furthermore, although professional modellers had clearly learnt, through years of practice, to cope with such problems when they occurred, their means of dealing with them using existing tools (e.g. having multiple viewports open, hiding parts of models, etc.) remained rather rudimentary, and not always very effective.

It is important to note that our studies mainly investigated focus and context awareness problems associated with 3D modelling tasks. These, however, are not the only types of problems faced by modellers in using 3D modelling software, and further research is clearly needed to study the extent of other challenges they deal with using such software.

Finally, it should also be noted that although our studies have been based on the use of polygonal modelling techniques, it is likely that other types of modelling techniques based on point cloud, solid geometry, etc. that require navigation and manipulation of points and objects in a virtual 3D world suffer from similar focus and context awareness problems identified here. Yet other techniques such as 3D scanning and sketching allow initial creation of 3D models, which usually need to be further manipulated and refined using polygonal modelling type techniques. As such, modellers using these types of tools would also end up facing focus and context awareness issues as part of their modelling process.

\section{ACKNOWLEDGEMENTS}

We would like to gratefully acknowledge the contributions of all our study participants, and Dr. Ray Littler for his assistance with statistical analysis of our study data. The user studies discussed in this article were approved by the Ethics Committee of the Faculty of Computing and Mathematical Sciences of the University of Waikato.

\section{REFERENCES}

Christopher Ahlberg and Ben Shneiderman. Visual information seeking: Tight coupling of dynamic query filters with starfield displays. In Proceedings of the SIGCHI Conference on Human Factors in Computing Systems, CHI '94, pages 313-317, New York, NY, USA, 1994. ACM. ISBN 0-89791650-6. doi: 10.1145/191666.191775.

Ferran Argelaguet and Carlos Andujar. Efficient 3d pointing selection in cluttered virtual environments. IEEE Computer Graphics and Applications, 29(6):34-43, 2009. ISSN 02721716. doi: 10.1109/MCG.2009.117.

Autodesk. http://usa.autodesk.com, 2014. visited in September 2014.

Ravin Balakrishnan. "beating" fitts' law: Virtual enhancements for pointing facilitation. International Journal of HumanComputer Studies, 61(6):857-874, December 2004. ISSN 1071-5819. doi: 10.1016/j.ijhcs.2004.09.002.

Patrick Baudisch and Carl Gutwin. Multiblending: Displaying 
MASOODIAN ET AL.

overlapping windows simultaneously without the drawbacks of alpha blending. In Proceedings of the SIGCHI Conference on Human Factors in Computing Systems, CHI '04, pages 367-374, New York, NY, USA, 2004. ACM. ISBN 1-58113702-8. doi: $10.1145 / 985692.985739$.

Patrick Baudisch, Nathaniel Good, Victoria Bellotti, and Pamela Schraedley. Keeping things in context: A comparative evaluation of focus plus context screens, overviews, and zooming. In Proceedings of the SIGCHI Conference on Human Factors in Computing Systems, CHI '02, pages 259266, New York, NY, USA, 2002. ACM. ISBN 1-58113-453-3. doi: $10.1145 / 503376.503423$.

Eric A. Bier, Maureen C. Stone, Ken Pier, William Buxton, and Tony D. DeRose. Toolglass and magic lenses: The see-through interface. In Proceedings of the 20th Annual Conference on Computer Graphics and Interactive Techniques, SIGGRAPH '93, pages 73-80, New York, NY, USA, 1993. ACM. ISBN 0-89791-601-8. doi: 10.1145/166117. 166126 .

Blender. http: //www . blender .org, 2014. visited in September 2014.

Doug A. Bowman, Ernst Kruijff, Joseph J. LaViola, and Ivan Poupyrev. An introduction to 3D user interface design. Presence: Teleoperators and Virtual Environments, 10(1): 96-108, February 2001. ISSN 1054-7460. doi: 10.1162/ 105474601750182342 .

Doug A. Bowman, Ernst Kruijff, Joseph J. LaViola, and Ivan Poupyrev. 3D User Interfaces: Theory and Practice. Addison Wesley Longman Publishing Co., Redwood City, CA, USA, 2004. ISBN 0201758679.

Virginia Braun and Victoria Clarke. Using thematic analysis in psychology. Qualitative Research in Psychology, 3(2):77-101, 2006. doi: 10.1191/1478088706qp063oa.

Stefan Bruckner, Peter Rautek, Ivan Viola, Mike Roberts, Mario Costa Sousa, and M. Eduard Gröllera. Hybrid visibility compositing and masking for illustrative rendering. Computers \& Graphics, 34(4):361-369, 2010. ISSN 00978493. doi: 10.1016/j.cag.2010.04.003.

Cynthia D. Bruyns, Steven Senger, Anil Menon, Kevin Montgomery, Simon Wildermuth, and Richard Boyle. A survey of interactive mesh-cutting techniques and a new method for implementing generalized interactive mesh cutting using virtual tools. The Journal of Visualization and Computer Animation, 13(1):21-42, 2002. doi: 10.1002/vis. 275 .

Marc Christie and Patrick Olivier. Camera control in computer graphics: Models, techniques and applications. In Proceedings of ACM Annual Conference on Computer Graphics and Interactive Techniques ASIA, SIGGRAPH ASIA '09, pages 3:1-3:197, New York, NY, USA, 2009. ACM. doi: $10.1145 / 1665817.1665820$.

Andy Cockburn, Amy Karlson, and Benjamin B. Bederson. A review of overview + detail, zooming, and focus + context interfaces. ACM Computing Surveys, 41(1):2:1-2:31, January 2008. ISSN 0360-0300. doi: 10.1145/1456650.1456652.
Donald A. Cox, Jasdeep S. Chugh, Carl Gutwin, and Saul Greenberg. The usability of transparent overview layers. In Cconference Summary on Human Factors in Computing Systems, CHI '98, pages 301-302, New York, NY, USA, 1998. ACM. ISBN 1-58113-028-7. doi: 10.1145/286498.286777.

Rudolph P. Darken and John L. Sibert. Wayfinding strategies and behaviors in large virtual worlds. In Proceedings of the SIGCHI Conference on Human Factors in Computing Systems, CHI '96, pages 142-149, New York, NY, USA, 1996. ACM. ISBN 0-89791-777-4. doi: 10.1145/238386.238459.

Ian Dey. Qualitative Data Analysis: A User Friendly Guide for Social Scientists. Routledge, 1993.

Joachim Diepstraten, Daniel Weiskopf, and Thomas Ertl. Interactive cutaway illustrations. Computer Graphics Forum, 22(3):523-532, 2003. ISSN 1467-8659. doi: 10.1111/1467-8659.t01-3-00700.

Doga. http://www.doga.co.jp/english/about/, 2014. visited in September 2014.

Paul Dourish. What we talk about when we talk about context. Personal Ubiquitous Computing, 8(1):19-30, February 2004. ISSN 1617-4909. doi: 10.1007/s00779-003-0253-8.

Paul Dourish and Victoria Bellotti. Awareness and coordination in shared workspaces. In Proceedings of the 1992 ACM Conference on Computer-supported Cooperative Work, CSCW '92, pages 107-114, New York, NY, USA, 1992. ACM. ISBN 0-89791-542-9. doi: 10.1145/143457.143468.

Niklas Elmqvist and Jean-Daniel Fekete. Semantic pointing for object picking in complex 3D environments. In Proceedings of Graphics Interface, GI '08, pages 243-250, Toronto, Ont., Canada, Canada, 2008. Canadian Information Processing Society. ISBN 978-1-56881-423-0.

Niklas Elmqvist, Ulf Assarsson, and Philippas Tsigas. Employing dynamic transparency for 3D occlusion management: Design issues and evaluation. In Cécilia Baranauskas, Philippe Palanque, Julio Abascal, and Simone Diniz Junqueira Barbosa publisher = IFIP International Federation for Information Processing, editors, Proceedings of the 11th IFIP TC 13 International Conference, INTERACT, volume 4662 of Lecture Notes in Computer Science, pages 532-545, 2007.

Niklas Elmqvist, Mihail Eduard Tudoreanu, and Philippas Tsigas. Evaluating motion constraints for 3D wayfinding in immersive and desktop virtual environments. In Proceedings of the SIGCHI Conference on Human Factors in Computing Systems, CHI '08, pages 1769-1778, New York, NY, USA, 2008. ACM. ISBN 978-1-60558-011-1. doi: 10.1145/1357054. 1357330

George Fitzmaurice, Justin Matejka, Igor Mordatch, Azam Khan, and Gordon Kurtenbach. Safe 3d navigation. In Proceedings of the Symposium on Interactive 3D Graphics and Games, I3D '08, pages 7-15. ACM, 2008. ISBN 978-159593-983-8. doi: 10.1145/1342250.1342252.

George W. Furnas. Generalized fisheye views. In Proceedings of the SIGCHI Conference on Human Factors in Computing 
Systems, CHI '86, pages 16-23, New York, NY, USA, 1986. ACM. ISBN 0-89791-180-6. doi: 10.1145/22627.22342.

George W. Furnas. A fisheye follow-up: Further reflections on focus + context. In Proceedings of the SIGCHI Conference on Human Factors in Computing Systems, CHI '06, pages 999-1008, New York, NY, USA, 2006. ACM. ISBN 1-59593372-7. doi: 10.1145/1124772.1124921.

G. E. Gorman and Peter Clayton. Qualitative Research For The Information Professional: A Practical Handbook. Facet Publications, 2nd edition, 2005. ISBN 978-1856044721.

Dimitris Grammenos, Alexandros Mourouzis, and Constantine Stephanidis. Virtual prints: Augmenting virtual environments with interactive personal marks. International Journal of Human-Computer Studies, 64(3):221-239, March 2006. ISSN 1071-5819. doi: 10.1016/j.ijhcs.2005.08.011.

Carl Gutwin. Improving focus targeting in interactive fisheye views. In Proceedings of the SIGCHI Conference on Human Factors in Computing Systems, CHI '02, pages 267-274, New York, NY, USA, 2002. ACM. ISBN 1-58113-453-3. doi: $10.1145 / 503376.503424$.

Carl Gutwin and Saul Greenberg. Design for individuals, design for groups: Tradeoffs between power and workspace awareness. In Proceedings of the 1998 ACM Conference on Computer Supported Cooperative Work, CSCW '98, pages 207-216, New York, NY, USA, 1998. ACM. ISBN 1-58113009-0. doi: 10.1145/289444.289495.

Carl Gutwin, Saul Greenberg, and Mark Roseman. Workspace awareness in real-time distributed groupware: Framework, widgets, and evaluation. In Proceedings of HCI on People and Computers XI, HCI '96, pages 281-298, London, UK, 1996. Springer-Verlag. ISBN 3-540-76069-5.

Chris Hand. A survey of 3D interaction techniques. Computer Graphics Forum, 16(5):269-281, 1997. ISSN 1467-8659. doi: 10.1111/1467-8659.00194.

Beverly L. Harrison, Gordon Kurtenbach, and Kim J. Vicente. An experimental evaluation of transparent user interface tools and information content. In Proceedings of the 8th Annual ACM Symposium on User Interface and Software Technology, UIST '95, pages 81-90, New York, NY, USA, 1995. ACM. ISBN 0-89791-709-X. doi: 10.1145/215585. 215669 .

Kasper Hornbæk, Benjamin B. Bederson, and Catherine Plaisant. Navigation patterns and usability of zoomable user interfaces with and without an overview. ACM Trans. Comput.-Hum. Interact., 9(4):362-389, December 2002. ISSN 1073-0516. doi: 10.1145/586081.586086.

Takeo Igarashi, Rieko Kadobayashi, Kenji Mase, and Hidehiko Tanaka. Path drawing for $3 \mathrm{D}$ walkthrough. In Proceedings of the 11th Annual ACM Symposium on User Interface Software and Technology, UIST '98, pages 173-174, New York, NY, USA, 1998. ACM. ISBN 1-58113-034-1. doi: $10.1145 / 288392.288599$.

Jacek Jankowski and Martin Hachet. A survey of interaction techniques for interactive $3 \mathrm{D}$ environments. In Proceedings of Eurographics - State of the Art Reports, Eurographics '13, pages 65-93. The Eurographics Association, 2013. doi: 10.2312/conf/EG2013/stars/065-093.

Azam Khan, Ben Komalo, Jos Stam, George Fitzmaurice, and Gordon Kurtenbach. Hovercam: Interactive 3D navigation for proximal object inspection. In Proceedings of the Symposium on Interactive 3D Graphics and Games, I3D '05, pages 73-80, New York, NY, USA, 2005. ACM. ISBN 159593-013-2. doi: 10.1145/1053427.1053439.

Azam Khan, Igor Mordatch, George Fitzmaurice, Justin Matejka, and Gordon Kurtenbach. Viewcube: A 3d orientation indicator and controller. In Proceedings of the Symposium on Interactive 3D Graphics and Games, I3D '08, pages 17-25, New York, NY, USA, 2008. ACM. ISBN 9781-59593-983-8. doi: 10.1145/1342250.1342253.

Mohamed Essam Khedr. An Infrastructure for Managing Context Information in Pervasive Computing Environments. University of Ottawa, 2004.

Sebastian Knödel, Martin Hachet, and Pascal Guitton. Interactive generation and modification of cutaway illustrations for polygonal models. In Andreas Butz, Brian Fisher, Marc Christie, Antonio Krüger, Patrick Olivier, and Roberto Therón, editors, Smart Graphics, volume 5531 of Lecture Notes in Computer Science, pages 140-151. Springer Berlin Heidelberg, 2009. ISBN 978-3-642-02114-5. doi: 10.1007/ 978-3-642-02115-2_12.

Robert Kosara, Silvia Miksch, and Helwig Hauser. Semantic depth of field. In Proceedings of the IEEE Symposium on Information Visualization, InfoVis 2001, pages 97-104, Los Alamitos, CA, USA, 2001. IEEE Computer Society. doi: 10.1109/INFVIS.2001.963286.

Robert Kosara, Silvia Miksch, and Helwig Hauser. Focus+context taken literally. IEEE Computer Graphics and Applications, 22(1):22-29, January 2002. ISSN 0272-1716. doi: 10.1109/38.974515.

Jens Krüger and Thomas Fogal. Focus and contextvisualization without the complexity. In Olaf Dössel and Wolfgang C. Schlegel, editors, Proceedings of the World Congress on Medical Physics and Biomedical Engineering, volume 25/13 of IFMBE '09, pages 45-48. Springer Berlin Heidelberg, 2010. ISBN 978-3-642-03894-5. doi: 10.1007/ 978-3-642-03895-2_14.

Jens Krüger, Jens Schneider, and Rüdiger Westermann. Clearview: An interactive context preserving hotspot visualization technique. IEEE Transactions on Visualization and Computer Graphics, 12(5):941-948, September 2006. ISSN 1077-2626. doi: 10.1109/TVCG.2006.124.

Arun Kulshreshth, Jonas Schild, and Joseph J. LaViola, Jr. Evaluating user performance in 3D stereo and motion enabled video games. In Proceedings of the International Conference on the Foundations of Digital Games, FDG '12, pages 33-40, New York, NY, USA, 2012. ACM. ISBN 9781-4503-1333-9. doi: 10.1145/2282338.2282350.

Jonathan Lazar, Jinjuan Heidi Feng, and Harry Hochheiser. Research Methods in Human-Computer Interaction. John 
Wiley \& Sons Ltd, 2010. ISBN 978-0-470-72337-1.

Y. K. Leung and Mark D. Apperley. A review and taxonomy of distortion-oriented presentation techniques. ACM Transactions on Computer-Human Interacteraction, 1(2):126-160, June 1994. ISSN 1073-0516. doi: 10.1145/ 180171.180173.

Wilmot Li, Lincoln Ritter, Maneesh Agrawala, Brian Curless, and David Salesin. Interactive cutaway illustrations of complex 3d models. ACM Transactions on Graphics, 26(3), July 2007. ISSN 0730-0301. doi: 10.1145/1276377.1276416.

Henry Lieberman. Powers of ten thousand: Navigating in large information spaces. In Proceedings of the rth Annual ACM Symposium on User Interface Software and Technology, UIST '94, pages 15-16, New York, NY, USA, 1994. ACM. ISBN 0-89791-657-3. doi: 10.1145/192426.192434.

Jock D. Mackinlay, Stuart K. Card, and George G. Robertson. Rapid controlled movement through a virtual 3D workspace. In Proceedings of the 17th Annual Conference on Computer Graphics and Interactive Techniques, SIGGRAPH '90, pages 171-176, New York, NY, USA, 1990. ACM. ISBN 0-89791344-2. doi: 10.1145/97879.97898.

Jock D. Mackinlay, George G. Robertson, and Stuart K. Card. The perspective wall: Detail and context smoothly integrated. In Proceedings of the SIGCHI Conference on Human Factors in Computing Systems, CHI '91, pages 173176, New York, NY, USA, 1991. ACM. ISBN 0-89791-383-3. doi: $10.1145 / 108844.108870$.

Masood Masoodian, Sam McKoy, Bill Rogers, and David Ware. DeepDocument: use of a multi-layered display to provide context awareness in text editing. In Proceedings of the working conference on Advanced visual interfaces, AVI '04, pages 235-239. ACM, 2004. ISBN 1-58113-867-9. doi: 10.1145/989863.989902. URL http://doi .acm.org/10.1145/989863.989902.

Michael J. McGuffin, Liviu Tancau, and Ravin Balakrishnan. Using deformations for browsing volumetric data. In Proceedings of IEEE Visualization, IEEE VIS 2003, pages 401-408, 2003. doi: 10.1109/VISUAL.2003.1250400.

Francisco Moura Pinto and CarlaMaria Dal Sasso Freitas. Illustrating volume data sets and layered models with importance-aware composition. The Visual Computer, 27(10):875-886, 2011. ISSN 0178-2789. doi: 10.1007/ s00371-011-0606-7.

Fabrizio Nunnari and Carla Simone. Perceiving awareness information through 3d representations. In Proceedings of the Working Conference on Advanced Visual Interfaces, AVI '04, pages 443-446, New York, NY, USA, 2004. ACM. ISBN 1-58113-867-9. doi: 10.1145/989863.989947.

Ji-Young Oh and Wolfgang Stuerzlinger. Moving objects with $2 \mathrm{~d}$ input devices in cad systems and desktop virtual environments. In Proceedings of Graphics Interface, GI '05, pages 195-202, School of Computer Science, University of Waterloo, Waterloo, Ontario, Canada, 2005. Canadian Human-Computer Communications Society. ISBN 1-56881$265-5$.
Ji-Young Oh, Wolfgang Stuerzlinger, and John Danahy. Sesame: Towards better 3d conceptual design systems. In Proceedings of the 6th Conference on Designing Interactive Systems, DIS '06, pages 80-89, New York, NY, USA, 2006. ACM. ISBN 1-59593-367-0. doi: 10.1145/1142405.1142419.

Ken Perlin and David Fox. Pad: An alternative approach to the computer interface. In Proceedings of the 20th Annual Conference on Computer Graphics and Interactive Techniques, SIGGRAPH '93, pages 57-64, New York, NY, USA, 1993. ACM. ISBN 0-89791-601-8. doi: 10.1145/166117. 166125 .

Pixologic. http://pixologic.com/zbrush/, 2014. visited in September 2014.

Catherine Plaisant, David Carr, and Ben Shneiderman. Imagebrowser taxonomy and guidelines for designers. IEEE Software, 12(2):21-32, 1995. ISSN 0740-7459. doi: 10.1109/ 52.368260 .

Thomas Porter and Tom Duff. Compositing digital images. In Proceedings of the 11th ACM Annual Conference on Computer Graphics and Interactive Techniques, SIGGRAPH '84, pages 253-259, New York, NY, USA, 1984. ACM. ISBN 0-89791-138-5. doi: 10.1145/800031.808606.

Ivan Poupyrev, Mark Billinghurst, Suzanne Weghorst, and Tadao Ichikawa. The Go-Go interaction technique: Nonlinear mapping for direct manipulation in VR. In Proceedings of the 9th Annual ACM Symposium on User Interface Software and Technology, UIST '96, pages 79-80, New York, NY, USA, 1996. ACM. ISBN 0-89791-798-7. doi: 10.1145/ 237091.237102.

PureDepth. http://www.puredepth.com, 2014. Retrieved in July 2014.

Gonzalo Ramos, George Robertson, Mary Czerwinski, Desney Tan, Patrick Baudisch, Ken Hinckley, and Maneesh Agrawala. Tumble! Splat! helping users access and manipulate occluded content in $2 \mathrm{~d}$ drawings. In Proceedings of the Working Conference on Advanced Visual Interfaces, AVI '06, pages 428-435, New York, NY, USA, 2006. ACM. ISBN 159593-353-0. doi: 10.1145/1133265.1133351.

C. Russo dos Santos, P. Gros, P. Abel, D. Loisel, N. Trichaud, and J. P. Paris. Metaphor-aware 3D navigation. In Proceedings of the IEEE Symposium on Information Visualization, InfoVis 2000, pages 155-165, 2000. doi: 10. 1109/INFVIS.2000.885104.

Doug Schaffer, Zhengping Zuo, Saul Greenberg, Lyn Bartram, John Dill, Shelli Dubs, and Mark Roseman. Navigating hierarchically clustered networks through fisheye and fullzoom methods. ACM Transactions on Computer-Human Interaction, 3(2):162-188, June 1996. ISSN 1073-0516. doi: $10.1145 / 230562.230577$.

Jonas Schild, Liane Bölicke, Joseph J. LaViola Jr., and Maic Masuch. Creating and analyzing stereoscopic 3D graphical user interfaces in digital games. In Proceedings of the SIGCHI Conference on Human Factors in Computing Systems, CHI '13, pages 169-178, New York, NY, USA, 2013. ACM. ISBN 978-1-4503-1899-0. doi: 10.1145/2470654. 
2470678 .

Bill Schilit, Norman Adams, and Roy Want. Context-aware computing applications. In Proceedings of the Workshop on Mobile Computing Systems and Applications, WMCSA '94, pages 85-90, Washington, DC, USA, 1994. IEEE Computer Society. ISBN 978-0-7695-3451-0. doi: 10.1109/MCSA.1994. 512740 .

Robert Spence and Mark Apperley. Data base navigation: An office environment for the professional. Behaviour $\mathbb{E}$ Information Technology, 1(1):43-54, 1982. doi: 10.1080/ 01449298208914435.

Desney S. Tan, George G. Robertson, and Mary Czerwinski. Exploring 3d navigation: Combining speed-coupled flying with orbiting. In Proceedings of the SIGCHI Conference on Human Factors in Computing Systems, CHI '01, pages 418425, New York, NY, USA, 2001. ACM. ISBN 1-58113-327-8. doi: $10.1145 / 365024.365307$.

Norman G. Vinson. Design guidelines for landmarks to support navigation in virtual environments. In Proceedings of the SIGCHI Conference on Human Factors in Computing Systems, CHI '99, pages 278-285, New York, NY, USA, 1999. ACM. ISBN 0-201-48559-1. doi: 10.1145/302979.303062.

Ivan Viola, Armin Kanitsar, and M. Eduard Gröller. Importance-driven feature enhancement in volume visualization. IEEE Transactions on Visualization and Computer Graphics, 11(4):408-418, 2005. ISSN 1077-2626. doi: 10.1109/TVCG.2005.62.

William B. L. Wong, Ronish Joyekurun, Hoda Mansour, Paola Amaldi, Anna Nees, and Rochelle Villanueva. Depth, layering and transparency: Developing design techniques. In Proceedings of the 17th Australia Conference on ComputerHuman Interaction: Citizens Online: Considerations for Today and the Future, OZCHI '05, pages 1-10, Narrabundah, Australia, Australia, 2005. Computer-Human Interaction Special Interest Group (CHISIG) of Australia. ISBN 159593-222-4.

Michelle Yeh and Christopher D. Wickens. Display signaling in augmented reality: Effects of cue reliability and image realism on attention allocation and trust calibration. Human Factors: The Journal of the Human Factors and Ergonomics Society, 43(3):355-365, 2001. doi: 10.1518/ 001872001775898269 .

Gary M. Yontef. Awareness, Dialogue and Process: Essays on Gestalt Therapy. The Gestalt Journal Press, Gouldsboro, ME, USA, 1993. ISBN 978-0939266203.

Shumin Zhai, William Buxton, and Paul Milgram. The "silk cursor": Investigating transparency for 3D target acquisition. In Proceedings of the SIGCHI Conference on Human Factors in Computing Systems, CHI '94, pages 459-464, New York, NY, USA, 1994. ACM. ISBN 0-89791-650-6. doi: 10.1145/ 191666.191822.

Stefanie Zollmann, Denis Kalkofen, Christof Hoppe, Stefan Kluckner, Horst Bischof, and Gerhard Reitmayr. Interactive $4 \mathrm{D}$ overview and detail visualization in augmented reality. In Proceedings of the IEEE International Symposium on Mixed and Augmented Reality, ISMAR '12, pages 167-176, Los Alamitos, CA, USA, 2012. IEEE Computer Society. ISBN 978-1-4673-4660-3. doi: 10.1109/ISMAR.2012.6402554.

\section{APPENDIX}


Table A1. A summary of the pilot study questionnaire.

\begin{tabular}{|c|c|}
\hline No. & Question and (answer range) \\
\hline 1 & Age range (below 20, 20-25, above 20) \\
\hline 2 & Gender (male, female) \\
\hline 3 & 3D modelling experience (3ds Max, Blender, Cinema 4 D, LightWave, Maya, Wing 3D) \\
\hline 4 & Do you feel you have successfully completed the assignment? (yes, no) \\
\hline 5 & $\begin{array}{l}\text { How often did you delete a 3D object you were working on and started with a new object? (never, one or } \\
\text { more times) }\end{array}$ \\
\hline 6 & $\begin{array}{l}\text { How regularly did you use/view each of the following viewport options? (In orthogonal mode: camera view, } \\
\text { front view, side view, top view, view all) (In perspective mode: camera view, front view, side view, top view, } \\
\text { view all) }\end{array}$ \\
\hline 7 & $\begin{array}{l}\text { If you have used the "view all" window, how important was it to see all the objects you had created? (not } \\
\text { important-very important) }\end{array}$ \\
\hline 8 & $\begin{array}{l}\text { Blender does not have an indicator for the eye location in its various views, how problematic was this when } \\
\text { finding your location in the } 3 \mathrm{D} \text { world? (not problematic-very problematic) }\end{array}$ \\
\hline 9 & $\begin{array}{l}\text { How easy was it to select a single vertex when there were many vertices in your model? (not difficult-very } \\
\text { difficult) }\end{array}$ \\
\hline 10 & $\begin{array}{l}\text { How easy was it to select a group of vertices (e.g. an edge or face) together when there were many edges or } \\
\text { faces in your model? (not difficult-very difficult) }\end{array}$ \\
\hline 11 & $\begin{array}{l}\text { How easy was it to align objects in perspective view (e.g. when putting an object on top of another object)? } \\
\text { (not easy-very easy) }\end{array}$ \\
\hline 12 & How useful would it be to show object names (labels) in perspective view? (not useful-very useful) \\
\hline \multirow[t]{2}{*}{13} & Do you know what the occlusion effect is? (yes, no) \\
\hline & If yes, how useful do you think it will be to have it? (not useful-ve \\
\hline 14 & Do you know what the parallax effect is? (yes, no) \\
\hline \multirow{2}{*}{15} & $\mathrm{D}$ \\
\hline & If yes, how useful do you think it will be to have it? (not useful-very useful) \\
\hline
\end{tabular}


IDENTIFYING PROBLEMS ASSOCIATED WITH FOCUS AND CONTEXT AWARENESS IN 3D MODELLING TASKS

Table A2. A summary of the interview questions.

\begin{tabular}{ll}
\hline No. & Question \\
\hline 1 & Can you tell me a bit about yourself and your experience with 3D modelling? \\
2 & What are the 3D modelling software you commonly use to create your models? \\
3 & What is one of the most challenging projects you have ever worked on? \\
4 & What is the average time required to complete a project? \\
5 & Do you do computer animation as well? \\
6 & How much time (in percentage terms) do you spend on modelling or animation? \\
7 & How do you compare the difficulty level of 3D modelling versus animation? \\
8 & Do you recycle or reuse your own existing models for new models, or do you always start from scratch? \\
9 & Have you ever used or improved someone else's model? \\
10 & If yes to 9: what has been your experience when working with or improving someone else's model? \\
11 & When you start a model, do you have everything clear in your mind or does it develop as you progress? \\
12 & For other elements such as lights, textures, color, etc., do you have everything clear in your mind when you \\
& start a model or does it develop as you progress?
\end{tabular}

13 For texturing, do you generally draw your textures from scratch or do you start with existing textures or reference photos?

14 What is the most common type of viewport you use when performing 3D modelling tasks?

15 When editing objects, do you prefer to work in orthographic or perspective mode?

16 How do you maintain position awareness when navigating in the 3D modelling scene; that is do you know what object you are looking at and from which angle?

17 Have you encountered a situation in which you are not sure what object you are looking at and from which angle?

18 If yes to 17: what caused it to happen and how did you resolve it?

19 When manipulating or transforming objects, what are the approaches that you take?

20 Have you encountered any situation or condition in which you have not been sure what you have done or the extent of manipulation that you applied to an object?

21 Have you had any experience of not knowing which side of an object you are looking at after manipulating or transforming the object?

22 How do you manage selecting objects, vertices, edges or faces of a model?

23 When editing a model (e.g. extruding, adding, deleting and grouping vertices, edges or faces) how do you make sure that you are selecting the correct component?

24 Have you encountered a situation in which you selected and edited the wrong object or component?

25 Do you have any difficulties recognizing the distance between front, internal or back parts of the model?

26 How do you verify which components are nearer to you?

27 For a single model, do you model several parts and then assemble them, or do you add patches to the edges of existing patches?

28 If several parts are modelled in 27: When assembling different parts, have you encountered any problems in realizing objects' relative position?

29 If several parts are modelled in 27: What are the methods that you use for assembling different parts?

30 What types of models or scenes require you to work in groups?

31 When working in a group, how many team members are involved in a single project and how are they classified?

32 Based on your experience, how is collaboration managed?

33 For all members in the group, how are the scale and texture of different objects determined? Is it determined by an individual?

34 What is the most common problem faced by members when working in a group?

35 Is there an individual team member assigned to do the final arrangement of objects in a 3D scene?

36 If yes to 35: Does that particular individual have any rights to amend or modify different objects?

37 If yes to 35: Is the process of placing $3 \mathrm{D}$ objects and stitching undertaken by that particular individual?

38 If yes to 35: What are the most common problems faced by that particular individual? 
Table A3. The study participants' modelling experience.

\begin{tabular}{lll}
\hline Participant & Years of experience & 3D Modelling software used \\
\hline 1 & 2 & MAYA, 3ds Max, Blender \\
2 & 7 & MAYA, 3ds Max \\
3 & 8 & MAYA \\
4 & 11 & MAYA, ZBrush \\
5 & 6 & MAYA, 3ds Max, Blender, ZBrush \\
6 & 5 & MAYA \\
7 & 5 & MAYA, 3ds Max, Blender \\
8 & 10 & MAYA, ZBrush \\
9 & 5 & MAYA, 3ds Max, ZBrush, Lightwave \\
10 & 6 & MAYA, 3ds Max \\
11 & 4 & MAYA, 3ds Max \\
12 & 8 & MAYA, 3ds Max, Softimage \\
13 & 5 & MAYA, 3ds Max, ZBrush \\
\hline
\end{tabular}

Table A4. A summary of the study participants' focus and context awareness problems. Blank cells indicate "no".

\begin{tabular}{llllr}
\hline Participant & $\begin{array}{l}\text { Maintaining posi- } \\
\text { tion awareness }\end{array}$ & $\begin{array}{l}\text { Identifying and } \\
\text { selecting objects } \\
\text { or components }\end{array}$ & $\begin{array}{l}\text { Recognizing the } \\
\text { distance between } \\
\text { objects or compo- } \\
\text { nents }\end{array}$ & $\begin{array}{l}\text { Realizing the rel- } \\
\text { ative position of } \\
\text { objects or compo- } \\
\text { nents }\end{array}$ \\
\hline 1 & yes & yes & yes & yes \\
2 & yes & yes & yes & yes \\
3 & yes & yes & yes & yes \\
4 & yes & yes & yes & \\
5 & yes & yes & yes & yes \\
6 & yes & yes & yes & yes \\
7 & yes & yes & yes & yes \\
8 & yes & & yes & yes \\
9 & & yes & yes \\
10 & yes & yes & yes & yes \\
11 & yes & yes & yes & yes \\
12 & yes & & &
\end{tabular}

